\title{
Out of the Dark and Into the Light: A New View of Phytochrome Photobodies
}

\author{
Sarah A. Pardi ${ }^{1,2}$ and Dmitri A. Nusinow ${ }^{1,2 *}$ \\ 'Donald Danforth Plant Science Center, St. Louis, MO, United States, ${ }^{2}$ Division of Biology and Biomedical Sciences, \\ Washington University in St. Louis, St. Louis, MO, United States
}

\section{OPEN ACCESS}

Edited by: Cornelia Klose,

University of Freiburg, Germany

Reviewed by:

Martina Legris,

University of Lausanne, Switzerland

Eirini Kaiserli,

University of Glasgow,

United Kingdom

*Correspondence:

Dmitri A. Nusinow

meter@danforthcenter.org

Specialty section:

This article was submitted to

Plant Cell Biology,

a section of the journal

Frontiers in Plant Science

Received: 29 June 2021 Accepted: 05 August 2021 Published: 31 August 2021

Citation:

Pardi SA and Nusinow DA (2021) Out of the Dark and Into the Light:

A New View of Phytochrome

Photobodies.

Front. Plant Sci. 12:732947. doi: 10.3389/fp/s.2021.732947
Light is a critical environmental stimulus for plants, serving as an energy source via photosynthesis and a signal for developmental programming. Plants perceive light through various light-responsive proteins, termed photoreceptors. Phytochromes are red-light photoreceptors that are highly conserved across kingdoms. In the model plant Arabidopsis thaliana, phytochrome B serves as a light and thermal sensor, mediating physiological processes such as seedling germination and establishment, hypocotyl growth, chlorophyll biogenesis, and flowering. In response to red light, phytochromes convert to a biologically active form, translocating from the cytoplasm into the nucleus and further compartmentalizes into subnuclear compartments termed photobodies. PhyB photobodies regulate phytochrome-mediated signaling and physiological outputs. However, photobody function, composition, and biogenesis remain undefined since their discovery. Based on photobody cellular dynamics and the properties of internal components, photobodies have been suggested to undergo liquid-liquid phase separation, a process by which some membraneless compartments form. Here, we explore photobodies as environmental sensors, examine the role of their protein constituents, and outline the biophysical perspective that photobodies may be undergoing liquid-liquid phase separation. Understanding the molecular, cellular, and biophysical processes that shape how plants perceive light will help in engineering improved sunlight capture and fitness of important crops.

\footnotetext{
Keywords: phytochrome, photobodies, biomolecular condensates, liquid-liquid phase separation, intrinsically disordered protein
}

\section{INTRODUCTION}

Light is the most critical environmental stimulus for all plant development, serving as the energy source for photosynthesis and as an environmental cue to regulate growth and development. Thus, it is critical for plants to appropriately detect, coordinate, and respond to light cues for their overall fitness and survival. To perceive light, plants have evolved different classes of photoreceptors that absorb light wavelengths from the UV to far-red (380-735 nm wavelengths), including UV RESISTANCE LOCUS 8 (UVR8), PHOTOTROPINS, CRYPTOCHROMES, LOV (Light, Oxygen, Voltage)-KELCH DOMAIN containing F-box proteins, and PHYTOCHROMES (Butler et al., 1959; Gressel, 1979; Kandori et al., 1992; Jansen et al., 1998; Briggs and Huala, 1999; Cashmore et al., 1999; Lin, 2000; Nelson et al., 2000; Somers et al., 2000; Briggs et al., 2001; Jarillo et al., 2001; 
Kinoshita et al., 2001; Kagawa et al., 2001; Sakai et al., 2001; Schultz et al., 2001; Briggs and Christie, 2002; Kasahara et al., 2002; Lin, 2002; Frohnmeyer and Staiger, 2003; Imaizumi et al., 2003; Kleine et al., 2003; Ulm and Nagy, 2005; Kaiserli and Jenkins, 2007; Quail, 2010; Yu et al., 2010; Chaves et al., 2011; Rizzini et al., 2011; Jenkins and Brown, 2018).

Phytochromes perceive red/far-red light $(600-750 \mathrm{~nm})$ and regulate many aspects of plant development, including seed germination, de-etiolation, gravitropism, flowering, circadian rhythms, and senescence (Bae and Choi, 2008; Franklin and Quail, 2010; Kami et al., 2010; Paik and Huq, 2019). In the model plant Arabidopsis thaliana, phytochromes are a fivemember family, phyA-phyE (Jones and Quail, 1989; Somers et al., 1991; Quail, 1991; López-Juez et al., 1992; Clack et al., 1994; Mathews, 2010). PhyA is classified as light-labile and is the most abundant phytochrome in etiolated seedlings, whereas phyB-E are classified as light-stable (Clack et al., 1994; Nagy and Schäfer, 2002). PhyA is mainly responsible for sensing and responding to far-red light, in addition to red light, whereas phyB-E are responsible for photomorphogenesis in response to red light and foliar shade (Whitelam et al., 1992; McCormac et al., 1993; Nagatani et al., 1993; Parks and Quail, 1993; Whitelam et al., 1993; Reed et al., 1994; Paik and Huq, 2019). Phytochrome A and $\mathrm{B}$ have overlapping and distinct photosensory roles in seedling development (Reed et al., 1994).

Phytochromes are dimeric chromoproteins, with each monomer covalently attached to a light-absorbing linear tetrapyrrole chromophore, phytochromobilin (Cornejo et al., 1992; Terry et al., 1995). The protein domains in plant phytochromes can be divided into two modules: the chromophore-bearing, N-terminal photosensory module, responsible for light perception and signaling, and the C-terminal module that directs nuclear localization, dimerization, and nuclear body formation (Rockwell et al., 2006; Nagatani, 2010). Phytochromes exist in two stable conformers: a biologically inactive red-light absorbing form (Pr) and a biologically active far-red light-absorbing form (Pfr) (Rockwell et al., 2006; Bae and Choi, 2008). The phytochrome holoprotein is assembled in the cytosol in the inactive Pr conformation. Once converted to Pfr in response to red light, phytochromes move from the cytoplasm into the nucleus, where most signaling functions occur (Sakamoto and Nagatani, 1996; Kircher et al., 1999, 2002; Yamaguchi et al., 1999; Kim et al., 2000).

Phytochrome function is dependent on its localization in the nucleus (Huq et al., 2003; Matsushita et al., 2003; Genoud et al., 2008). Phytochrome A-E have differing mechanisms by which they are transported to the nucleus. Of the phytochromes, phyA nuclear localization is well characterized and is dependent on FAR-RED ELONGATED HYPOCOTYL 1 (FHY1) and FHY1LIKE (FHL) (Hiltbrunner et al., 2006; Genoud et al., 2008; Helizon et al., 2018). FHY1 and FHL act as shuttle proteins, binding to phyA-Pfr in the cytoplasm and transporting it to the nucleus (Hiltbrunner et al., 2006; Genoud et al., 2008; Rausenberger et al., 2010; Helizon et al., 2018). An NLS-like motif in the C-terminal domain of phyB-E is sufficient for localizing these phytochromes to the nucleus (Sakamoto and Nagatani, 1996; Chen et al., 2005), and the C-terminal module is necessary for nuclear localization (Matsushita et al., 2003; Chen et al., 2005). PHYTOCHROME INTERACTING FACTOR 3 (PIF3) (Pfeiffer et al., 2012) and SUPPRESSOR OF PHYA-105 (SPA1) (Zheng et al., 2013) have been shown to promote nuclear import of phyB-Pfr. However, the transport mechanism remains to be defined experimentally. PhyB nuclear localization may involve complementary binding partners under varying light conditions. Nuclear localization of phyC, phyD, and phyE remain the least understood (Ádám et al., 2013; Klose et al., 2015b). Future studies are required to define which proteins, if any, are not only sufficient but necessary for transporting phyB-E.

Phytochrome signaling is responsive to temperature in addition to light, since the Pfr form is thermally unstable. Phytochromes undergo isomerization from the active Pfr form to the inactive Pr state in a light-independent, temperaturedependent process called dark or thermal reversion (Jung et al., 2016; Legris et al., 2016). (For a review on phytochrome thermal reversion, readers are pointed to Klose et al., 2020). Two studies showed that phytochromes serve as temperature sensors through their thermal reversion capability. Using genomic approaches, Jung et al. demonstrated that phytochromes alter the Arabidopsis transcriptome in response to warm temperatures (Jung et al., 2016). Specifically, temperature affects phyB's ability to bind to target genes' promoters and repress PIF4 activity. PIFs are antagonists of phytochromes, promoting hypocotyl growth (Ni et al., 1998; Oh et al., 2007; Shen et al., 2008; Lorrain et al., 2009; Shin et al., 2009; Leivar and Quail, 2011). Additionally, they showed that phyB's dark reversion integrates temperature signals during the night (Jung et al., 2016). Legris et al. (2016) demonstrated in a complementary study - through genetics, biochemical measurement of phyB Pfr:Pr isomerization, and modeling approaches - that increased temperature reduces the amount of phyB Pfr pool and strength of signaling. In addition, a negative correlation was shown between temperature and phyB activity. Overall, these two breakthrough studies concluded that in addition to functioning as a light sensor, phyB is a thermosensor in plants (Jung et al., 2016; Legris et al., 2016), providing critical mechanistic insight into how plants perceive warm temperatures. This insight shapes future research on light signaling to mitigate the harmful effects of increasing global temperature on agriculturally important crops.

A critical step in phytochrome signaling is the assembly of active phytochrome Pfr into large (>500 nm) subnuclear membraneless compartments termed photobodies (Yamaguchi et al., 1999; Chen et al., 2003; Huang et al., 2016). Increasing the intensity of red light, which stabilizes the Pfr form, promotes the formation of large photobodies (Chen et al., 2003; Van Buskirk et al., 2012). Conversely, conditions that revert Pfr to Pr, such as far-red light, high temperature, or darkness, cause photobodies to disassemble into many smaller foci and ultimately dissipate within the nucleoplasm (Kircher et al., 1999; Yamaguchi et al., 1999; Chen and Chory, 2011; Van Buskirk et al., 2012, 2014). Photobody localization is conserved in dicots and monocots (Kircher et al., 1999, 2002; Kim et al., 2000). Recent work supports that photobodies are an essential cellular structure for phyB signaling (Su and Lagarias, 2007; Chen et al., 2010; Kaiserli et al., 2015; Huang et al., 2016, 2019; Enderle et al., 2017; 
Qiu et al., 2017; Yang et al., 2019; Yoo C.Y. et al., 2019). There are several hypothesized functions of photobodies, and multiple proteins have been shown to colocalize with photobodies (Holm et al., 2002; Bauer et al., 2004; Subramanian et al., 2004; Al-Sady et al., 2006; Hiltbrunner et al., 2006; Jang et al., 2007; Yu et al., 2008; Liu et al., 2011; Yan et al., 2011; Zuo et al., 2011; Van Buskirk et al., 2012). However, photobody function, protein components, and biogenesis are yet to be clearly defined.

Below, we explore the biological significance of photobodies in Arabidopsis thaliana, how photobodies contribute to signaling in fluctuating environments, protein components that promote photobody formation, photobody biogenesis, how liquidliquid phase separation (LLPS) may underlie the biophysical mechanism of assembly, and photobody functions. Lastly, we discuss some of the many exciting directions for future research on LLPS of photobodies.

\section{DISCOVERY AND BIOLOGICAL SIGNIFICANCE}

Subnuclear structures of phytochrome were first observed in 1999 in transgenic plants overexpressing phyB-GFP (Yamaguchi et al., 1999). Different nomenclature has been used throughout the years to describe these subnuclear compartments: speckles, foci, nuclear bodies, and ultimately photobodies. Using immunoblotting and various microscopy tools, Yamaguchi et al. reported the light-dependent subcellular distribution of phyB-GFP. This fusion protein localized to the nucleus and further compartmentalized into foci with a size of $\sim 1 \mu \mathrm{m}$. They also observed a positive correlation between the duration of red-light exposure and the size of the phyB-GFP photobody. The authors compared these phyB nuclear bodies to promyelocytic leukemia (PML) bodies, which are involved in RNA metabolism and transcription regulation (Stuurman et al., 1990; LallemandBreitenbach, 2010). This was the first description connecting phyB photobodies to nuclear condensates found in other systems. Since this fundamental study used a highly active Cauliflower Mosaic Virus 35S promoter to express phyB-GFP, it was suggested that the existence of these structures was merely due to overexpression (Yamaguchi et al., 1999). However, electron microscopy experiments using immuno-gold labeling of phyA in Arabidopsis demonstrated that endogenous phytochromes formed photobodies (Sheerin et al., 2015). Further work found that all members of the phy family formed nuclear bodies at differing rates in response to red and white light (Kircher et al., 2002), suggesting that photobody localization was a regulated process. Thus, the subnuclear assembly of phyB-GFP is presumed to reflect endogenous phyB localization (Yamaguchi et al., 1999; Gil et al., 2000; Kim et al., 2000; Kircher et al., 2002).

Even though these studies demonstrated that photobodies are not artifacts of exogenous expression, their biological importance remained in question. Matsushita et al. overexpressed phyB's N-terminal domain fused to a nuclear localization sequence (NLS) and a dimerization domain (Matsushita et al., 2003). They found that this phyB chimera did not form photobodies but was sufficient for phyB signaling, rescuing several phyB physiological responses in constant redlight conditions (Matsushita et al., 2003). Thus, the authors concluded that nuclear localization, not photobody formation, was necessary for phyB signaling (Matsushita et al., 2003). Furthermore, under low red-light conditions where phyB is dispersed throughout the nucleoplasm rather than localized to photobodies, plants show physiological responses reflecting the presence of active phyB. Thus, nucleoplasmic phyB is sufficient for signaling in these conditions (Parks and Quail, 1993; Wagner et al., 1996; Gil et al., 2000; Krall and Reed, 2000; Chen et al., 2003). It is possible that photobodies may promote certain phyB functions but not others, or that photobodies may act to enhance phyB activity through its N-terminal module. In agreement with this, Chen et al. (2003) proposed a model in which phyB is active both when dispersed throughout the nucleoplasm and when localized to photobodies, with different phyB mechanisms responding to varying intensities of red light.

Although the studies above argue that photobodies are dispensable, accumulating evidence supports the idea that photobodies are essential for phyB signaling, as discussed below. PhyB-GFP mutants that are nuclear-localized but do not form nuclear bodies have impaired light signaling, demonstrating a strong correlation between phyB biological activity and compartmentalization into photobodies (Su and Lagarias, 2007; Chen et al., 2010; Kaiserli et al., 2015; Huang et al., 2016, 2019; Enderle et al., 2017; Qiu et al., 2017; Yang et al., 2019; Yoo C.Y. et al., 2019). Although Matsushita et al. (2003) showed that photobodies are dispensable in constant red light, a study by Van Buskirk et al. (2014) demonstrated the role of photobodies under more natural light/dark conditions. Using PhyB mutants, a strong link was shown between photobodies and phyB regulated processes after dusk. Specifically, the presence of photobodies was highly correlated with hypocotyl elongation inhibition in the dark and PIF3 degradation (Van Buskirk et al., 2014). During the day, phytochromes promote photomorphogenesis and inhibit hypocotyl elongation by repressing PIF activity via proteasome-mediated degradation (Al-Sady et al., 2006; Leivar et al., 2009; Shin et al., 2009; Leivar and Quail, 2011). When photobodies disassemble, PIF3 is no longer repressed, and hypocotyl growth is promoted (Van Buskirk et al., 2014). In agreement, Qiu et al. (2017) demonstrated that expression of phyB's C-terminal module in stable transgenic lines forms photobodies and is sufficient for degrading PIF3 and regulating a subset of PIF-dependent genes. Challenging a previous model in which phyB's N-terminal module was responsible for PIF3's lightinduced degradation, they demonstrated that PIF3's degradation is dependent on its interaction with the dimeric C-terminal module (Shimizu-Sato et al., 2002; Oka et al., 2008; Kikis et al., 2009; Leivar and Quail, 2011; Qiu et al., 2017). Additionally, the significance of photobodies can be seen at the phenotypic level, with phyB photobody localization patterns being tightly correlated with the degree of hypocotyl elongation. Pfr, which promotes the formation of large photobodies, produces a short hypocotyl phenotype, whereas when photobodies are small or dispersed in low red:far-red (R:FR) light, seedlings are taller (Chen et al., 2003). Overall, there is growing acceptance that 
photobodies are essential for phyB responses (Kircher et al., 2002; Chen et al., 2003; Oka et al., 2008).

\section{PHOTOBODIES ARE IMPORTANT FOR ENVIRONMENTAL SENSING AND SIGNALING}

Photobody formation is responsive to the external environment, as is described below. They are found to be important for light, circadian, and temperature signaling, potentially acting as hubs connecting these signaling pathways and serving as environmental sensors in plants (Kircher et al., 2002; Chen et al., 2003; Huang et al., 2019; Hahm et al., 2020; Figure 1).

\section{Light Signaling}

Photobody formation responds to specific wavelengths and intensities of light (Kircher et al., 1999; Yamaguchi et al., 1999; Kim et al., 2000; Kircher et al., 2002; Chen et al., 2003). The amount of phyB-Pfr to total phyB and the formation of photobodies depends on the intensity of red light and the ratio of R:FR. The size and distribution patterns of photobodies correlates with the intensity of red light. As red light intensity increases from $0.5 \mu \mathrm{mol} \cdot \mathrm{m}^{-2} \cdot \mathrm{sec}^{-1}$ to $8 \mu \mathrm{mol} \cdot \mathrm{m}^{-2} \cdot \mathrm{sec}^{-1}$, PhyB-GFP is first evenly dispersed within the nucleoplasm, then hundreds of small photobodies form, then large photobodies $(\sim 1 \mu \mathrm{m})$ appear along with the small photobodies, and finally, large photobodies exclusively form within the nucleus. Thus, the increase in light intensity in a dosage dependent manner and the resulting photoconversion and nuclear localization of Pfr correlates with the appearance of large photobodies (Chen et al., 2003). Within minutes of initial light exposure, phyA and phyB rapidly assemble into many small early 'transient' bodies, but after extended light exposure, a few large 'stable' bodies form, mainly made up of phyB (Casal et al., 2002; Kircher et al., 2002). These larger, stable bodies are referred to as 'photobodies,' which are correlated with functional phyA and phyB activity (Kircher et al., 1999, 2002; Yamaguchi et al., 1999; Kim et al., 2000). Under red light, PIF3 transiently colocalizes with these early phyB photobodies, where it then gets degraded in a light-dependent manner (Bauer et al., 2004). In the biogenesis of photobodies, it is likely that early transient bodies merge into the larger stable photobodies. Overall, the amount of phyB-Pfr to total phyB, controlled by red light, has to surpass a critical threshold to form photobodies (Chen et al., 2003).

Changing R:FR ratios, responsible for shade avoidance responses, has the same effect on photobody formation as light intensity (Casal et al., 2002; Chen et al., 2003; Trupkin et al., 2014). Low R:FR reduces phyB-Pfr to total phyB, which reduces the number of large photobodies and leads to the formation of many small photobodies (Trupkin et al., 2014). This pattern is reversible-when plants are transferred back from either low to high irradiance or R:FR, large photobodies form, demonstrating their dynamic behavior (Trupkin et al., 2014). Overall, these nuclear bodies may potentially act as light sensors, forming and dissipating in response to specific wavelengths and intensities of light.

\section{Circadian Regulation}

The circadian clock may regulate photobody dynamics. In agreement with being light-responsive, photobody accumulation increases over the day (Kircher et al., 2002). Surprisingly, when grown under short-day conditions ( $8 \mathrm{~h}$ light:16 h dark), phyA, phyB, phyC, and phyE-GFP nuclear body number increased significantly before dawn in anticipation of the subjective light period (Kircher et al., 2002). Based on these findings, it was

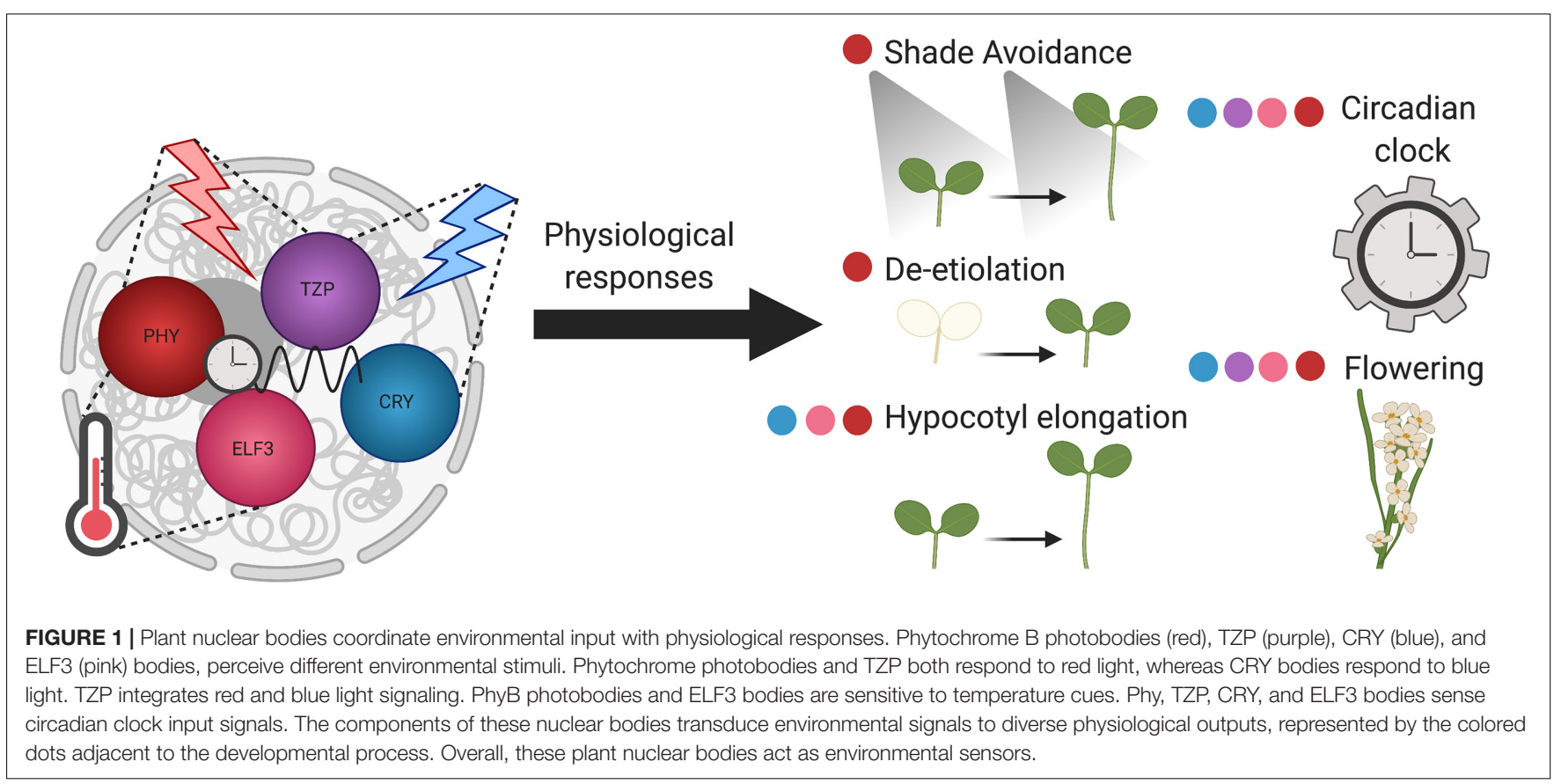


concluded that photobodies are modulated by the circadian clock (Kircher et al., 2002). However, it remains unclear what mechanism allows for the circadian-regulated dispersal and reassembly of phy-Pfr photobodies.

In addition to being regulated by the clock, phytochromes also signal into the plant circadian clock (Somers et al., 1998; Martínez-García et al., 2000; Hu et al., 2013). A recent study demonstrated that photobodies are important for the entrainment of the circadian clock oscillator (Huang et al., 2019). This study utilized the phyB constitutively active mutant phyB ${ }^{\mathrm{Y} 276 \mathrm{H}}$ (YHB) to specifically activate phyB signaling while keeping other photoreceptors turned off in the dark (Hu et al., 2009). YHB is sufficient for maintaining circadian oscillations of a Luciferase reporter under darkness, a condition that typically leads to dampening of clock rhythms in wild-type plants (Jones, 2009). When YHB is present in a mutant background that prevents photobody formation, YHB's constitutively photomorphogenic phenotype and light input into the circadian clock are abolished (Huang et al., 2019). Specifically, this line without photobodies could not sustain circadian rhythms in constant darkness, even though the $\mathrm{YHB}$ allele locked phyB in its active state (Huang et al., 2019). Together, these studies demonstrate that photobody dynamics are both an input and output of the plant circadian clock.

\section{Temperature Perception}

Not only do photobodies sense light and circadian cues, photobodies also act as temperature sensors through phyB's thermal reversion ability (Jung et al., 2016; Legris et al., 2016). Thermal reversion causes the pool of Pfr to be reduced in warmer temperatures and thus decreases the size of photobodies (Legris et al., 2016). In response to temperature, photobodies within a nucleus can vary in localization patterns, stabilities, and tissue-specific dynamics (Hahm et al., 2020). Individual photobodies have distinct thermostabilities; in response to warm temperatures, thermosensitive photobodies rapidly disassemble, while thermo-insensitive photobodies remain unaltered (Hahm et al., 2020). Hahm et al. (2020) also found that some photobodies are located adjacent to the nucleolus, termed nucleolar associated photobodies, while others were found distributed throughout the nucleus, termed non-nucleolar associated photobodies. The non-nucleolar associated photobodies were found to be thermosensitive, while the nucleolar associated photobodies were thermo-insensitive, suggesting a connection between thermostability and photobody position within the nucleus (Hahm et al., 2020). Another recent study provided evidence that increasing temperatures decreases the size of photobodies during the night, and that phyB can transfer night-time temperature information to influence the next day's hypocotyl growth (Murcia et al., 2020). Lastly, genetic evidence demonstrated that the hypocotyl growth of Arabidopsis lacking photobodies was hypersensitive to high temperature under long days, suggesting photobodies affect thermoresponsiveness (Huang et al., 2019). These studies provide strong evidence that photobody assembly and disassembly are highly responsive to fluctuating temperatures. In sum, photobodies are important for sensing and responding to diverse environmental cues, particularly light, circadian, and temperature signals (Figure 1).

\section{PROTEIN COMPONENTS REGULATING PHOTOBODY FORMATION}

There are multiple protein components thought to make up photobodies, many of which are involved in light signaling through gene regulation or proteolysis (Holm et al., 2002; Bauer et al., 2004; Subramanian et al., 2004; Al-Sady et al., 2006; Hiltbrunner et al., 2006; Jang et al., 2007; Yu et al., 2008; Liu et al., 2011; Yan et al., 2011; Zuo et al., 2011; Van Buskirk et al., 2012). However, the term 'photobody' does not necessarily encompass all the various potential components into a single entity. There are likely distinct nuclear bodies forming in response to different environmental stimuli. While many proteins colocalize to photobodies, thus far, there are only a few that are shown to regulate phyB-photobody formation.

HEMERA (HMR), also known as pTAC12, is involved in proteolysis and transcription and was the first protein component to be identified as essential for photobody formation (Chen et al., 2010; Nevarez et al., 2017). The hmr mutant has an albino and long hypocotyl phenotype, indicating its involvement in chloroplast biogenesis and red light signaling. Ultimately, HMR couples nuclear and chloroplastic gene expression (Chen et al., 2010). HMR localizes in chloroplasts, functioning as an essential plastid-encoded plastid RNA polymerase (PEP) component and as an activator of PEP-mediated plastid-encoded photosynthetic genes. HMR then relocates into the nucleus where it modulates phy-PIF signaling (Pfalz et al., 2006; Chen et al., 2010; Steiner et al., 2011; Nevarez et al., 2017). The hmr mutant displays phyB photobodies that are either smaller in size or phyB-GFP dispersed throughout the nucleoplasm, demonstrating that HMR promotes phyB photobody formation (Chen et al., 2010). HMR is not only essential for photobody formation but is also required for phyA, PIF1, and PIF3 degradation (Chen et al., 2010; Galvão et al., 2012). The binding of phyB Pfr with HMR promotes the accumulation of HMR protein, which is required for PIF3 degradation in the dark (Galvão et al., 2012). In addition to mediating PIF1 and PIF3 protein degradation, HMR alters PIFs regulation of target genes (Galvão et al., 2012). Specifically, HMR directly interacts with PIF1 and PIF3 as a transcriptional coactivator to regulate a class of PIF target genes (Qiu et al., 2015). These studies propose that photobodies are sites for proteasomal degradation and provide evidence that supports the tight correlation between photobody formation and phyB function (Chen et al., 2010).

The second protein identified as essential for phyB photobody formation was PHOTOPERIODIC CONTROL OF HYPOCOTYL 1 (PCH1). PCH1 and its homolog PCH1-LIKE (PCHL) directly bind to phyB, phyD, and phyE, preferentially interacting with the Pfr form (Huang et al., 2016; Enderle et al., 2017). Under short-day conditions, pch1 Arabidopsis seedlings have an elongated hypocotyl phenotype, similar to phyB's elongated hypocotyl phenotype (Huang et al., 2016). PCH1 and PCHL inhibit and slow phyB thermal reversion in vivo and 
in vitro, stabilizing phyB-Pfr (Enderle et al., 2017; Huang et al., 2019). The constitutively active phyB mutant, YHB, showed that $\mathrm{PCH} 1$ is essential for phyB photobody formation and serves as a structural component in vivo (Huang et al., 2019). While the overexpression of PCHL increases photobody formation, PCHL is not required for photobody formation. In the $p c h 1$ phyB-GFP mutant, either one photobody is formed or phyB-GFP signal is dispersed within the nucleus. Both HMR and $\mathrm{PCH} 1$ are involved in temperature sensing, with HMR being required for phyB daytime temperature sensing through PIF4 (Huang et al., 2019; Qiu et al., 2019). Affinity Purification Mass Spectrometry (APMS) data of $\mathrm{PCH} 1$ protein interactions shows an overlap of $\mathrm{PCH} 1$ interacting partners with other photobody components, such as phyA-E, CONSTITUTIVE PHOTOMORPHOGENIC 1 (COP1), SPA1, EARLY FLOWERING 3 (ELF3), and TANDEM ZINCFINGER PLUS3 (TZP), suggesting PCH1 and PCH1-interacting proteins are bona fide photobody components (Huang et al., 2016). Interestingly, PCH1 co-immunoprecipitation data did not identify $\mathrm{HMR}$ as an interacting partner, suggesting PCH1-mediated phyB photobodies may be distinct from HMR-mediated phyB photobodies (Huang et al., 2016).

In two recent studies, the paralogs REGULATOR OF CHLOROPLAST BIOGENESIS (RCB) and NUCLEAR CONTROL OF PEP ACTIVITY (NCP) were identified in a forward genetic screen as plastid and nuclear-localized proteins, similar to HMR. Importantly, they were shown to regulate phyB photobody biogenesis (Yang et al., 2019; Yoo C.Y. et al., 2019). Similar to HMR, RCB, and NCP first localize to plastids, then translocate to the nucleus where they participate in phyB signaling. Furthermore, RCB and NCP also promote the formation of large photobodies and the degradation of PIF1 and PIF3 in the nucleus (Yoo C.Y. et al., 2019). Similar to $h m r$ phyB-GFP, the $r c b$ phyB-GFP and the ncp phyB-GFP mutants cannot assemble into large photobodies, but instead form many smaller nuclear bodies (Yang et al., 2019; Yoo C.Y. et al., 2019). RCB is involved in the same phyB pathway as HMR, binds to Pfr and Pr of phyA and phyB, and modulates chloroplast biogenesis via PIF degradation (Yoo C.Y. et al., 2019). While NCP plays an essential role in chloroplast biogenesis by regulating PEP assembly, RCB is not required for chloroplast biogenesis (Yang et al., 2019). These two studies proposed a model which connects phyB photobody biogenesis in the nucleus to plastid anterograde signaling, ultimately connecting photomorphogenesis with de-etiolation (Yang et al., 2019; Yoo C.Y. et al., 2019). The functions of photobody components support the importance of photobodies in phy-PIF mediated physiological responses.

In addition to phytochromes forming nuclear bodies, some phytochrome interacting proteins are sufficient for forming nuclear bodies. One example is TZP, which forms nuclear bodies in a red light and phyB-dependent manner (Kaiserli et al., 2015; Figure 1). These TZP-phyB bodies are dynamic, diurnally regulated, and associate with RNA (Kaiserli et al., 2015). TZP's association with RNA infers that these bodies may be involved in RNA metabolism, a function of many nuclear bodies that contain RNA binding proteins (reviewed in Sabari et al., 2020). Interestingly, TZP-phyB bodies do not colocalize with HMR (Kaiserli et al., 2015). Thus, similar to HMR-phyB photobodies being distinct from PCH1-phyB photobodies, HMR-phyB photobodies may be separate from TZP-phyB photobodies.

Circadian clock proteins, such as ELF3 and GIGANTEA (GI), and proteins involved in light signaling, such as CRYPTOCHROME 1 (CRY1), CRY2, and E3 ubiquitin ligase COP1, also form nuclear bodies (Ang et al., 1998; Wang et al., 2001; Yu et al., 2009; Kim et al., 2013; Figure 1). ELF3, a member of the Arabidopsis EVENING COMPLEX and interactor of phyB, transmits light and temperature signals to the circadian clock, potentially through its ability to form nuclear bodies (Jung et al., 2020). ELF3 nuclear bodies colocalize with TZP, suggesting their connection in circadian signaling (Kaiserli et al., 2015). CRY1 and CRY2 form nuclear bodies in response to blue light intensity (Yu et al., 2009; Gu et al., 2012; Figure 1). For further reading on other examples of plant nuclear bodies, readers are pointed to the following excellent reviews (Ronald and Davis, 2019; Emenecker et al., 2020; Meyer, 2020). Based on their protein components, phyB photobodies, and other nuclear bodies, may have both shared and distinct functions.

\section{PHOTOBODY BIOGENESIS}

Another long-standing question is the mechanism by which photobodies form. However, studies directly testing photobody biogenesis are limited. Thus far, synthetic biology and mathematical modeling have been the main approaches taken in elucidating their association.

The first study to assess how photobodies form utilized a nucleolus-tethering system (NoTS) (Liu et al., 2014). They designed their NoTS based on the bacterial LacO/LacI tethering system used previously to study nuclear body assembly in mammalian cells (Kaiser et al., 2008; Mao et al., 2011; Shevtsov and Dundr, 2011; Dundr, 2013). The NoTS artificially tethers a protein of interest to the nucleolus by fusion with a nucleolus marker protein (Liu et al., 2014). This allows the visualization of components localizing to and initiating nuclear body formation (Liu et al., 2014). They showed that these tethered photobodies are functional and found that the efficiency of phyB to initiate photobody formation was lower than that of other proteins, such as COP1, CRY1, and CRY2 (Liu et al., 2014). The lower efficiency of phyB to initiate assembly suggests that other factors assist phyB in photobody formation (Chen et al., 2010; Huang et al., 2019; Yang et al., 2019; Yoo C.Y. et al., 2019). Overall, this study concluded that since multiple photobody-localized proteins are sufficient to form nuclear bodies, photobodies form via self-organization (Liu et al., 2014).

Mathematical modeling has been another approach in understanding how photobodies form. Previous studies used mathematical modeling to assess the effect of phyB-Pfr conformation and dimerization on photobody association and dissolution (Rausenberger et al., 2010; Klose et al., 2015a). One study used mathematical modeling and statistical physics to understand the mechanism of phyB photobody biogenesis (Grima et al., 2018). Although their model assumed that photobodies are solely made up of phyB dimers, which is not the 
case in vivo (Van Buskirk et al., 2012), their calculations suggested that the kinetics of building photobodies could not simply reflect the assembly phyB dimers (Grima et al., 2018). Therefore, they reasoned that photobody formation consists of two steps: a fast nucleation step in which phyB aggregates or binds to other proteins, followed by a slower step of more complex binding. Grima et al. (2018) also suggested that photobodies may be hollow, which has since been supported by microscopy evidence (Perrella et al., 2021). In this case, phyB may bind to a structural component, also referred to as a seed component, to initiate formation (Mao et al., 2011; Shevtsov and Dundr, 2011; Figure 2). Since phytochromes homodimerize and heterodimerize with each other (Sharrock and Clack, 2004), phyB may first form small aggregates, followed by the slower process of more complex associations with other proteins and or nucleic acids (Figure 2). Although there are a few studies on photobody formation utilizing synthetic biology and mathematical modeling, another approach to study this cellular process may be through the lens of biophysics.

\section{PHOTOBODIES MAY UNDERGO LIQUID-LIQUID PHASE SEPARATION}

Photobodies have been described as plant-specific biomolecular condensates (Cuevas-Velazquez and Dinneny, 2018; Emenecker et al., 2020). Biomolecular condensates are subcellular, membraneless compartments that concentrate biomolecules, such as proteins and nucleic acids, to organize cellular processes (Spector, 2006; Banani et al., 2017). Although terminology varies for these membraneless compartments, biomolecular condensate is a general term to describe the assembly of biomolecules, regardless of material properties or function (Banani et al., 2017). Many examples connect biomolecular condensates to neurodegenerative diseases, such as Alzheimer's, amyotrophic lateral sclerosis (ALS), Huntington's, and certain cancers (Spannl et al., 2019). Thus, understanding their biogenesis is

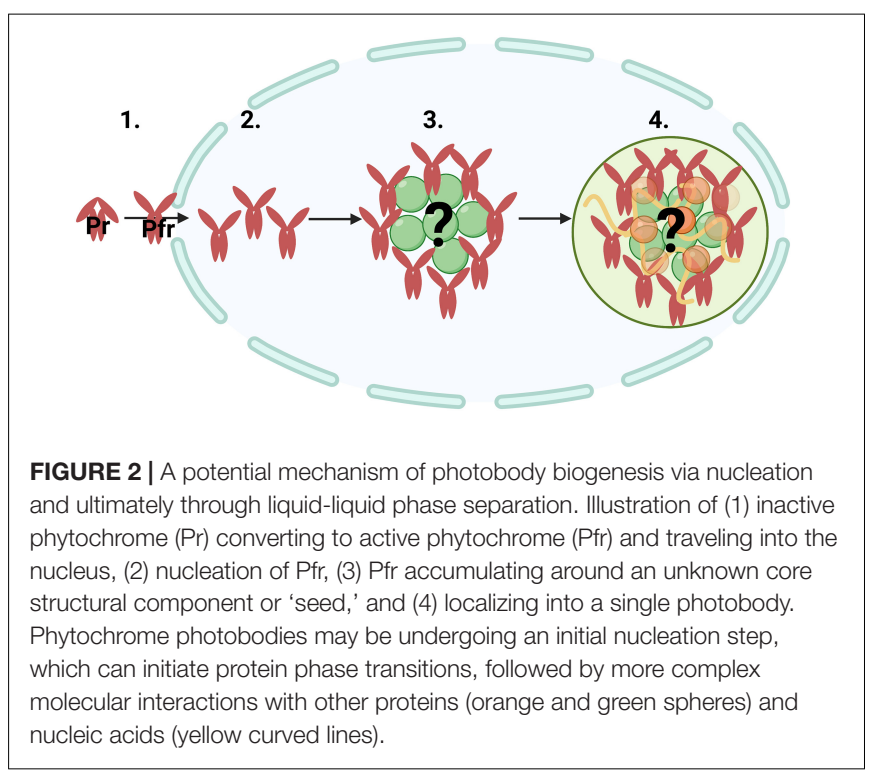

of significance in the medical field. Studies on the assembly of biomolecular condensates in fungal and mammalian systems are extensive but are less developed in plant systems.

A biophysical process by which condensates can form is through LLPS (Hyman et al., 2014). LLPS is the biophysical process by which two distinct liquid phases are formed, or demixed, into a dense phase and a less dense phase (Boeynaems et al., 2018; Posey et al., 2018; Gomes and Shorter, 2019; Figure 3). The key characteristics of condensates undergoing LLPS are that they display liquid-like properties: they are spherical, can fuse and relax like liquid droplets, and are dynamic - able to rapidly exchange with the surrounding cellular environment (Alberti et al., 2019). Previous fluorescence recovery after photobleaching (FRAP) experiments on phyB-YFP demonstrate that phyB photobodies are, in fact, dynamic (Rausenberger et al., 2010). Biomolecular condensates do not necessarily need to be liquidlike, they can be gel-like or take on a solid material state depending on the properties of the resident molecules.

Proteins that promote phase separation are typically multivalent and frequently contain intrinsically disordered regions (IDRs) or prion-like domains (Figure 4; Banani et al., 2017). Multivalent molecules can undergo inter- or intra- molecular interactions, binding to multiple partners simultaneously (Harmon et al., 2017). It is important to note that multivalent interactions drive phase separation, not necessarily intrinsically disordered proteins (IDPs) (Martin and Holehouse, 2020). Phytochrome's N-terminal extension (NTE) contains a predicted IDR, which may promote their condensation into photobodies (Burgie et al., 2021). Several proteins that colocalize to phyB photobodies are predicted to contain prion-like domains or IDRs, such as ELF3 and HY5 (Covarrubias et al., 2017; Cuevas-Velazquez and Dinneny, 2018; Jung et al., 2020). Proteins with IDRs in plant cells seem to play a role in different mechanisms underlying responses to environmental stimuli (Covarrubias et al., 2017).

The presence and concentration of key protein components contribute to the phase separation of condensates. As the abundance of nuclear Pfr increases with increasing red light intensity in a dosage dependent manner, large photobodies form, thus red light may promote the phase separation of photobodies in vivo (Figure 3). Conversely, as warm temperatures lead to the thermal reversion of Pfr back to Pr and the dissipation of photobodies, temperature may also regulate the phase separation of photobodies in vivo (Figure 3). Models that have been used to describe biomolecular condensate formation are the "stickers and spacers" model and the "scaffold and client" model (Semenov and Rubinstein, 1998; Banani et al., 2017; Posey et al., 2018; Wang A. et al., 2018; Holehouse, 2019). In the former model, stickers are portions of an amino acid sequence that promote intermolecular interactions, while the spacers are regions that are inert in promoting interactions (Wang A. et al., 2018). In the later model, scaffold proteins are necessary for condensate formation to occur, whereas client proteins localize to the condensate but are not necessary or sufficient for formation (Banani et al., 2017). In the context of photobodies, it may be helpful to think of HMR, PCH1, RCB, or NPC as 'scaffold' proteins, as they are essential for proper photobody formation (Figure 3). In contrast, 


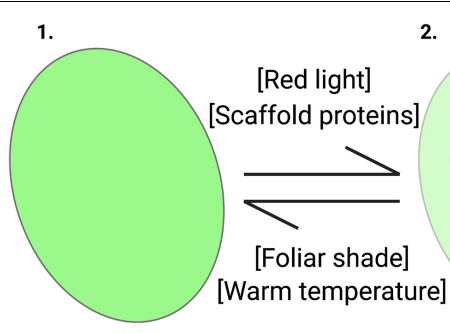

.

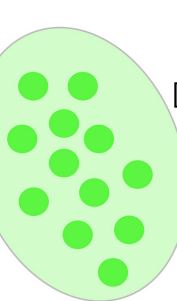

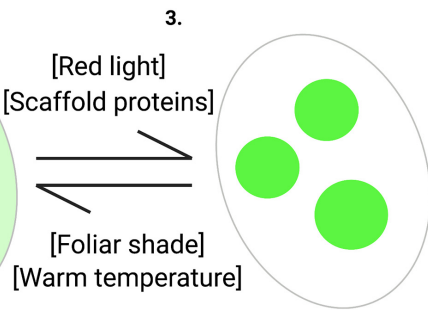

FIGURE 3 | Phytochrome photobodies are a controllable system for studying factors that influence liquid-liquid phase separation in vivo. Within Arabidopsis nuclei (ovals), active phytochrome can transition from a single diffuse phase throughout the nucleoplasm (1) into two distinct phases: one with a low concentration that is evenly distributed throughout the system and a denser phase that takes the shape of liquid droplets, or photobodies (2), and finally into large photobodies (3). Green represents nuclear phy-Pfr, and the green circles represent phytochrome photobodies. Environmental and cellular factors influence the formation and dissipation of phytochrome photobodies. Increasing red-light intensity and the concentration of presumptive scaffold proteins, such as HMR, PCH1, RCB, and NCP, promote the formation of photobodies. Conversely, warm temperatures and low red:far-red ratios under foliar shade conditions promote the dissipation of photobodies.

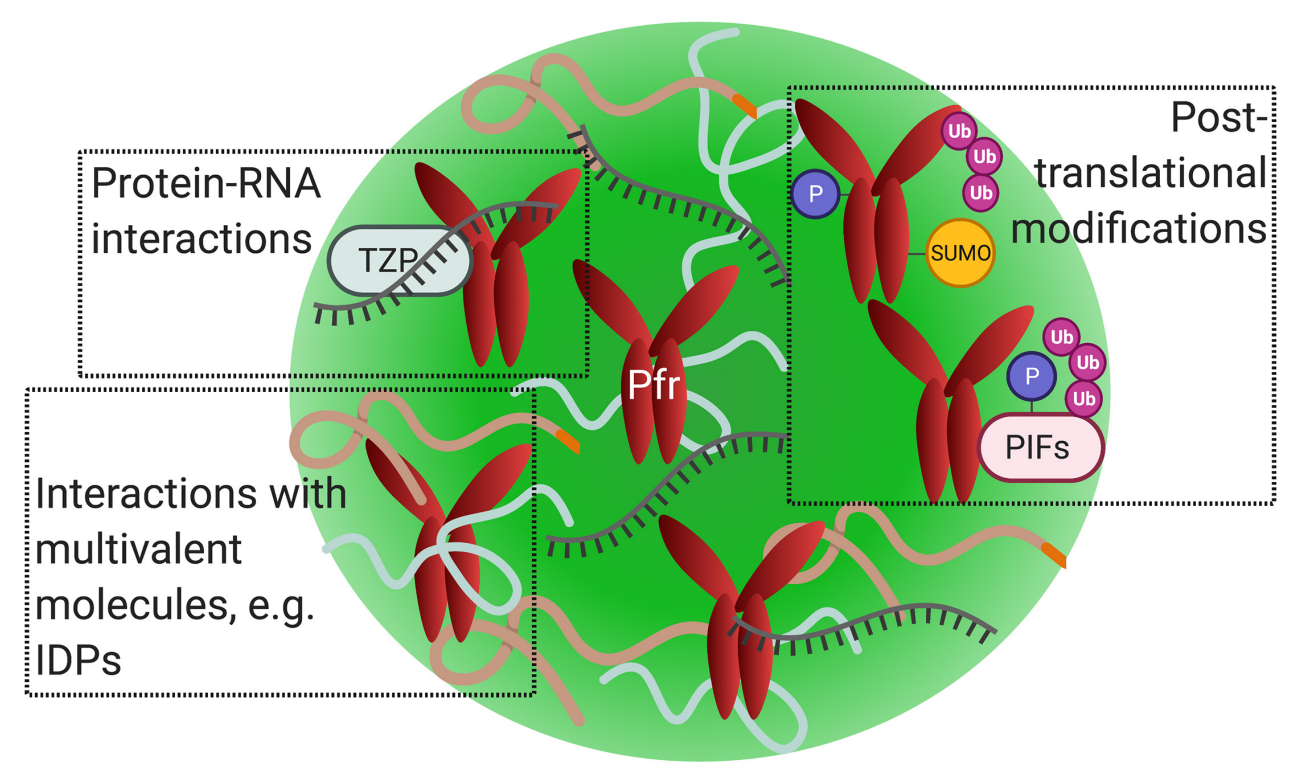

FIGURE 4 | Phytochrome photobodies may share features associated with other nuclear biomolecular condensates. Illustration of active phytochrome (Pfr) within a single photobody (green sphere). Factors that promote nuclear condensate formation via liquid-liquid phase separation, including protein-protein interactions, protein-nucleic acid interactions, and posttranslational modifications, may also function in photobodies. (Top left) Phytochrome photobodies may contain nucleic acids, as demonstrated by the co-localization of RNA with TZP-phyB nuclear bodies. (Top right) Phytochromes undergo posttranslational modifications such as SUMOylation, phosphorylation, and polyubiquination. Phytochromes target PIFs for phosphorylation and polyubiquitination. (Bottom left) Multivalent molecules, such as intrinsically disordered proteins (curved lines), may promote photobody formation.

the proteins that are shown to colocalize but are not necessary for formation may be thought of as 'clients'. Other factors, such as $\mathrm{pH}$, temperature, salinity, and environmental factors, also contribute to phase separation and formation of condensates (Yoo H. et al., 2019).

In addition, posttranslational modifications, such as SUMOylation, phosphorylation, and ubiquitination, are found to be associated with condensate formation or dissolution (reviewed in Owen and Shewmaker, 2019). Phytochromes are SUMOylated, phosphorylated, and ubiquitinated (Quail et al., 1978; Kim et al., 2004; Jang et al., 2010; Sadanandom et al., 2015; Figure 4). SUMOylation is shown to modulate far-red light-induced phyA signaling (Qu et al., 2020). PhyB is SUMOylated at its C-terminal module, which is enhanced by red light (Sadanandom et al., 2015). Since phyB's C-terminal module is required for photobody formation, there may be a potential connection between this posttranslational modification and photobodies. (For a review on plant SUMOylation, readers are pointed to reviews by Augustine and Vierstra, 2018; Srivastava et al., 2021). Phosphorylation of phytochromes at serine residues attenuates light signaling and interrupts proteinprotein interactions with downstream partners (reviewed in Hoang et al., 2019). For example, phosphorylation of phyB ${ }^{\text {Ser86 }}$ accelerates its thermal reversion and inhibits binding with PIF3 (Medzihradszky et al., 2013). The reversion of Pfr back to $\mathrm{Pr}$ in thermal reversion leads to the disassembly of photobodies; therefore, phyB's phosphorylation may be connected to photobody disassembly. Furthermore, nuclear 
phytochrome is targeted for polyubiquitination by COP1 in a red light and PIF mediated manner (Jang et al., 2010). Like other biomolecular condensates, the posttranslational modifications of phytochromes or binding partners could potentially be associated with photobody formation or dissipation (Figure 4).

Biomolecular condensates undergoing LLPS is an emerging topic in the plant field, and there are a growing number of studies on plant biomolecular condensates demonstrating LLPS (Fang et al., 2019; Powers et al., 2019; Dorone et al., 2020; Jung et al., 2020; Ouyang et al., 2020; Zavaliev et al., 2020; Huang et al., 2021). One of these recent findings was on a phyB interacting protein, ELF3, demonstrating that ELF3 nuclear bodies form via LLPS (Jung et al., 2020). Through biochemical and phenotypic analysis, Jung et al. (2020) demonstrated that the Arabidopsis ELF3 prion-like domain is necessary and sufficient for its temperature responsive phase separating ability in vivo and in vitro. ELF3's prion-like domain was shown to regulate thermoresponsive binding of ELF3 to target genes and flowering time (Jung et al., 2020). LLPS may be the mode by which ELF3 connects temperature signals with the circadian clock (Jung et al., 2020; Wilkinson and Strader, 2020). The field is just beginning to understand how plant biomolecular condensates form. Overall, there is a whole realm of exciting questions awaiting to be answered regarding the formation process of plant biomolecular condensates.

\section{PHOTOBODY FUNCTIONS}

The functions of nuclear condensates include, but are not limited to, protein/nucleic acid sequestration, protein ubiquitination, transcription regulation, RNA processing, and organizing genome structure (Sabari et al., 2020). To date, the function of photobodies has yet to be clearly defined. However, their hypothesized functions overlap with functions associated with other nuclear condensates, including protein storage, proteolysis, protein sequestration, and gene regulation (Van Buskirk et al., 2012).

The first hypothesized function is that nuclear bodies act as storage sites to stabilize Pfr and prevent phyB from reverting back to Pr (Rausenberger et al., 2010; Van Buskirk et al., 2014). In agreement with this, $\mathrm{PCH} 1$, one of the essential components of photobodies, stabilizes phyB-Pfr, slowing its thermal reversion rate and stabilizing phyB photobodies in the dark (Huang et al., 2016, 2019; Enderle et al., 2017). It is likely that being a storage center for active phyB is not the only role that photobodies play.

There is a strong line of evidence for the second hypothesized function that photobodies are sites for protein degradation. HMR is an essential component of photobodies and is critical for phyA, PIF1, and PIF3 degradation (Chen et al., 2010; Galvão et al., 2012). Light induces phyA and phyB's negative regulation of PIFs through phosphorylation, polyubiquitination, and 26S proteasomal degradation, possibly within nuclear bodies (Al-Sady et al., 2006; Lorrain et al., 2008; Dong et al., 2017). Conversely, PIF3 promotes the degradation of phyB (Ni et al., 2013). Light-Response-Bric-a-Brack/Tamtrack/Broad (LRB1/2/3) promotes the polyubiquitination of phyB and PIF3 in a light-dependent manner (Christians et al., 2012; Ni et al., 2014). PIF3 enhances LRB2 binding to phyB-Pfr, which is required for proteasome-mediated phyB protein degradation (Christians et al., 2012). This negative regulatory mechanism was termed the mutually assured destruction model. In this model, phyB stimulates the phosphorylation of PIF3, promoting the binding to LRBs, which leads to the polyubiquitination of both phyB and PIF3, targeting them for $26 \mathrm{~S}$ proteasomal degradation in a lightdependent manner (Ni et al., 2014). This bidirectional feedback loop of PIF3 and phyB proteolysis may occur within photobodies since their co-localization into early bodies is associated with PIF3 degradation (Bauer et al., 2004). Similarly, blue lightinduced CRY2 nuclear bodies are involved in degradation via its association with the $26 \mathrm{~S}$ proteasome (Shalitin et al., 2003; Yu et al., 2007, Yu et al., 2010; Wang et al., 2015; Liu et al., 2017). Overall, these light-mediated nuclear bodies may be sites for proteasomal degradation in light signaling.

A third hypothesized function of photobodies is to sequester proteins to modulate signaling. PhyA and phyB colocalize with SPA1 into nuclear bodies, which are important for the phyASPA1 interaction (Lu et al., 2015). Light activation of phyA nuclear bodies prevents COP1-SPA1-mediated degradation of LONG HYPOCOTYL IN FAR-RED 1 (HFR1), a promoter of photomorphogenesis (Sheerin et al., 2015). Similar to phyA bodies and their ability to block COP1-SPA1 activity, CRY1 co-localizes and directly interacts with SPA1 in CRY-bodies, attenuating the COP1-SPA1 interaction and negatively regulating COP1, albeit in a blue light-dependent manner (Lian et al., 2011; Zuo et al., 2011).

Lastly, there is evidence that photobodies may be involved in gene regulation. PhyB has been shown to regulate transcription, alternative splicing, and alternative promoter selection to mediate light responses, which could take place within photobodies (Shikata et al., 2014; Ushijima et al., 2017; Dong et al., 2020). Splicing factors, transcription factors, and transcriptional regulators are shown to colocalize to phyB-photobodies, suggesting a potential overlap of transcriptional and posttranscriptional regulation within these subnuclear compartments (Van Buskirk et al., 2012; Xin et al., 2017). One compelling example of photobodies demonstrating transcriptional activity are TZP-phyB bodies. TZP-phyB nuclear bodies are shown to serve as sites of active transcription, activating gene expression to promote flowering (Kaiserli et al., 2015). TZP is an RNA binding protein, and TZP-phyB bodies are shown to associate with RNA (Kaiserli et al., 2015; Figure 4). There are many examples of biomolecular condensates associating with nucleic acids, particularly RNA, such as paraspeckles and $\mathrm{P}$ granules (reviewed in Roden and Gladfelter, 2021). Since many condensates containing RNA binding proteins are involved in RNA processing, TZP bodies may also be involved in RNA metabolism. Similarly, blue light-induced CRY nuclear bodies are associated with transcription regulation (Wang Q. et al., 2018).

As there is supporting evidence for all hypothesized functions, including sites for storage, proteolysis, protein sequestration, and transcription regulation, photobodies likely have multiple functions within the cell to shape plant responses to various environmental stimuli. 


\section{RESEARCH DIRECTIONS AND OUTSTANDING QUESTIONS}

There has been a recent explosion of excitement and interest in biomolecular condensates across kingdoms. The idea that photobodies may be undergoing LLPS is not new (CuevasVelazquez and Dinneny, 2018). However, the idea that phase separation of biomolecular condensates connects cellular signaling in plants with the external environment is novel.

Phytochrome photobodies may serve as a controllable system to study LLPS in vivo and in vitro through its reversible formation and dissipation in response to environmental and cellular factors (Figure 3). Optogenetic tools use light to tightly control molecular and cellular signaling (Taslimi et al., 2014; Dine and Toettcher, 2018; Goglia and Toettcher, 2019). A recent optogenetic tool named OptoDropletTFs uses the Arabidopsis photoreceptors CRY2 or phyB, through their lightinduced oligomerization, as a molecular switch to induce LLPS (Schneider et al., 2021). Through the red light-inducible phyB-PIF6 interaction, this study made a phytochrome-based OptoDropletTF system by creating a phyB-IDR fusion protein to significantly increase transcriptional output via LLPS (Schneider et al., 2021). IDR-mediated phase separation may be a common mechanism in regulating transcription (Boija et al., 2018). As phyA, phyB, and PIFs are recruited to promoters to regulate transcription, LLPS may be occurring via the formation of photobodies to enhance these gene regulatory processes (Chen et al., 2010; Guo et al., 2012; Pfeiffer et al., 2012; Jung et al., 2016; Brodsky et al., 2020). Overall, photobodies may provide a useful system in studying LLPS to regulate cellular processes.

Photobody function, composition, and biogenesis have yet to be clearly defined since their discovery in 1999. Photobodies may be central points for regulating, organizing, and tightly coordinating the complex interception of phytochrome-mediated processes: photomorphogenesis, de-etiolation, flowering, etc. There is likely not just a single type of 'photobody', but rather numerous nuclear bodies that organize the complex overlapping of light, temperature, and circadian signaling pathways. Investigating the molecular, cellular, and biophysical properties that lead to the formation of these nuclear condensates will provide great insight into a potentially conserved mechanism by which nuclear condensates form. A combination of high-resolution microscopy, proteomic, genomic, structural, computational, and biophysical approaches will begin to answer questions such as: How do phytochrome photobodies form? What is their function? What is their composition? And can they

\section{REFERENCES}

Ádám, É, Kircher, S., Liu, P., Mérai, Z., González-Schain, N., Hörner, M., et al. (2013). Comparative functional analysis of full-length and $\mathrm{N}$-terminal fragments of phytochrome C, D and E in red light-induced signaling. New Phytol. 200, 86-96. doi: 10.1111/nph.12364

Alberti, S., Gladfelter, A., and Mittag, T. (2019). Considerations and challenges in studying liquid-liquid phase separation and biomolecular condensates. Cell 176, 419-434. doi: 10.1016/j.cell.2018.12.035 be manipulated to improve crop fitness? It will be very exciting to see what new information is discovered in the coming years.

There are boundless avenues for future research to further explore and characterize photobody biomolecular condensates through the lens of LLPS. Below are questions and topics that will be of interest:

- How can our understanding of photobodies and their role as environmental sensors be used to improve crops through altering shade, temperature, and daylength sensitivity?

- Are there multiple different types of photobodies present simultaneously in a single nucleus?

- Is there a physical interaction between nucleolar associated photobodies and the nucleolus?

- Do nucleolar associated photobodies share commonalities with Cajal bodies, which frequently associate with the nucleolus (Monneron and Bernhard, 1969; TrinkleMulcahy and Sleeman, 2017)?

- Does LLPS occur in vivo during phyB and PIF transcription regulation?

- Are there other nuclear bodies that associate with nucleic acids? If so, are target genomic loci recruited to photobodies for transcription?

- Are posttranslational modifications associated with photobody formation or dissipation?

- Can the effects of photobodies seen so far be recapitulated with phyB expression at endogenous levels?

\section{AUTHOR CONTRIBUTIONS}

SP and DN wrote the manuscript. Both authors contributed to the article and approved the submitted version.

\section{FUNDING}

This research was supported by the U.S. Department of Energy, Office of Science, Office of Biological and Environmental Research, Genomic Science Program grant no. DE-SC0021286.

\section{ACKNOWLEDGMENTS}

We would like to thank Elizabeth Haswell, Margaret Wilson, Nathan Swyers, Rebecca Bindbeutel, and Maria Sorkin for the thoughtful reading of this review and their helpful edits.
Al-Sady, B., Ni, W., Kircher, S., Scha fer, E., and Quail, P. H. (2006). Photoactivated phytochrome induces rapid PIF3 phosphorylation prior to proteasomemediated degradation. Mol. Cell. 23, 439-446. doi: 10.1016/j.molcel.2006.06. 011

Ang, L. H., Chattopadhyay, S., Wei, N., Oyama, T., Okada, K., Batschauer, A., et al. (1998). Molecular interaction between COP1 and HY5 defines a regulatory switch for light control of Arabidopsis development. Mol. Cell 1, 213-222. doi: 10.1016/s1097-2765(00)80 $022-2$ 
Augustine, R. C., and Vierstra, R. D. (2018). SUMOylation: rewiring the plant nucleus during stress and development. Curr. Opin. Plant Biol. 45, 143-154. doi: 10.1016/j.pbi.2018. 06.006

Bae, G., and Choi, G. (2008). Decoding of light signals by plant phytochromes and their interacting proteins. Annu. Rev. Plant Biol. 59, 281-311. doi: 10.1146/ annurev.arplant.59.032607.092859

Banani, S. F., Lee, H. O., Hyman, A. A., and Rosen, M. K. (2017). Biomolecular condensates: organizers of cellular biochemistry. Nat. Rev. Mol. Cell Biol. 18, 285-298. doi: 10.1038/nrm.2017.7

Bauer, D., Viczia’n, A., Kircher, S., Nobis, T., Nitschke, R., Kunkel, T., et al. (2004). Constitutive photomor- phogenesis 1 and multiple photoreceptors control degradation of phy- tochrome interacting factor 3 , a transcription factor required for light signaling in Arabidopsis. Plant Cell 16, 1433-1445. doi: $10.1105 /$ tpc. 021568

Boeynaems, S., Alberti, S., Fawzi, N. L., Mittag, T., Polymenidou, M., Rousseau, F., et al. (2018). Protein phase separation: a new phase in cell biology. Trends Cell Biol. 28, 420-435. doi: 10.1016/j.tcb.2018.02.004

Boija, A., Klein, I. A., Sabari, B. R., Dall'Agnese, A., Coffey, E. L., Zamudio, A. V., et al. (2018). Transcription factors activate genes through the phase-separation capacity of their activation domains. Cell 175, 1842-1855. doi: 10.1016/j.cell. 2018.10.042

Briggs, W. R., Beck, C. F., Cashmore, A. R., Christie, J. M., Hughes, J., Jarillo, J. A., et al. (2001). The phototropin family of photoreceptors. Plant Cell 13, 993-997. doi: $10.2307 / 3871358$

Briggs, W. R., and Christie, J. M. (2002). Phototropins 1 and 2: versatile plant blue- light receptors. Trends Plant Sci. 7, 204-210. doi: 10.1016/s1360-1385(02) 02245-8

Briggs, W. R., and Huala, E. (1999). Blue-light photoreceptors in higher plants. Annu. Rev. Cell Dev. Biol. 15, 33-62. doi: 10.1146/annurev.cellbio.15.1.33

Brodsky, S., Jana, T., Mittelman, K., Chapal, M., Kumar, D. K., Carmi, M., et al. (2020). Intrinsically disordered regions direct transcription factor in vivo binding specificity. Mol. Cell 79, 459-471. doi: 10.1016/j.molcel.2020.05.032

Burgie, E. S., Gannam, Z. T., McLoughlin, K. E., Sherman, C. D., Holehouse, A. S., Stankey, R. J., et al. (2021). Differing biophysical properties underpin the unique signaling potentials within the plant phytochrome photoreceptor families. Proc. Natl. Acad. Sci. 118:e2105649118. doi: 10.1073/pnas.2105649118

Butler, W. L., Norris, K. H., Siegelman, H. W., and Hendricks, S. B. (1959). Detection, assay, and preliminary purification of the pigment controlling photoresponsive development of plants. Proc. Natl. Acad. Sci. U. S. A. 45:1703. doi: 10.1073 /pnas.45.12.1703

Casal, J. J., Davis, S. J., Kirchenbauer, D., Viczian, A., Yanovsky, M. J., Clough, R. C., et al. (2002). The serine-rich N-terminal domain of oat phytochrome A helps regulate light responses and subnuclear localization of the photoreceptor. Plant Physiol. 129, 1127-1137. doi: 10.1104/pp.010977

Cashmore, A. R., Jarillo, J. A., Wu, Y. J., and Liu, D. (1999). Cryptochromes: blue light receptors for plants and animals. Science 284, 760-765. doi: 10.1126/ science.284.5415.760

Chaves, I., Pokorny, R., Byrdin, M., Hoang, N., Ritz, T., Brettel, K., et al. (2011). The cryptochromes: blue light photoreceptors in plants and animals. Annu. Rev. Plant Biol. 62, 335-364. doi: 10.1146/annurev-arplant-042110-103759

Chen, M., and Chory, J. (2011). Phytochrome signaling mechanisms and the control of plant development. Trends Cell Biol. 21, 664-671. doi: 10.1016/j.tcb. 2011.07.002

Chen, M., Galvão, R. M., Li, M., Burger, B., Bugea, J., Bolado, J., et al. (2010). Arabidopsis HEMERA/pTAC12 initiates photomorphogenesis by phytochromes. Cell 141, 1230-1240. doi: 10.1016/j.cell.2010.05.007

Chen, M., Schwab, R., and Chory, J. (2003). Characterization of the requirements for localization of phytochrome B to nuclear bodies. Proc. Natl. Acad. Sci. 100, 14493-14498. doi: 10.1073/pnas.1935989100

Chen, M., Tao, Y., Lim, J., Shaw, A., and Chory, J. (2005). Regulation of phytochrome B nuclear localization through light-dependent unmasking of nuclear-localization signals. Curr. Biol. 15, 637-642. doi: 10.1016/j.cub.2005. 02.028

Christians, M. J., Gingerich, D. J., Hua, Z., Lauer, T. D., and Vierstra, R. D. (2012). The light-response BTB1 and BTB2 proteins assemble nuclear ubiquitin ligases that modify phytochrome B and D signaling in Arabidopsis. Plant Physiol. 160, 118-134. doi: 10.1104/pp.112.199109
Clack, T., Mathews, S., and Sharrock, R. A. (1994). The phytochrome apoprotein family inArabidopsis is encoded by five genes: the sequences and expression ofPHYD andPHYE. Plant Mol. Biol. 25, 413-427. doi: 10.1007/bf0004 3870

Cornejo, J., Beale, S. I., Terry, M. J., and Lagarias, J. C. (1992). Phytochrome assembly. The structure and biological activity of 2 (R), 3 (E)-phytochromobilin derived from phycobiliproteins. J. Biol. Chem. 267, 14790-14798. doi: 10.1016/ s0021-9258(18)42109-6

Covarrubias, A. A., Cuevas-Velazquez, C. L., Romero-Pérez, P. S., Rendón-Luna, D. F., and Chater, C. C. (2017). Structural disorder in plant proteins: where plasticity meets sessility. Cell. Mol. Life Sci. 74, 3119-3147. doi: 10.1007/s00018$017-2557-2$

Cuevas-Velazquez, C. L., and Dinneny, J. R. (2018). Organization out of disorder: liquid-liquid phase separation in plants. Curr. Opin. Plant Biol. 45, 68-74. doi: 10.1016/j.pbi.2018.05.005

Dine, E., and Toettcher, J. E. (2018). Optogenetic reconstitution for determining the form and function of membraneless organelles. Biochemistry 57, 2432-2436. doi: 10.1021/acs.biochem.7b01173

Dong, J., Chen, H., Deng, X. W., Irish, V. F., and Wei, N. (2020). Phytochrome $B$ induces intron retention and translational inhibition of PHYTOCHROMEINTERACTING FACTOR3. Plant Physiol. 182, 159-166. doi: 10.1104/pp.19. 00835

Dong, J., Ni, W., Yu, R., Deng, X. W., Chen, H., and Wei, N. (2017). Lightdependent degradation of PIF3 by SCFEBF1/2 promotes a photomorphogenic response in Arabidopsis. Curr. Biol. 27, 2420-2430. doi: 10.1016/j.cub.2017. 06.062

Dorone, Y., Boeynaems, S., Jin, B., Bossi, F., Flores, E., Lazarus, E., et al. (2020). Hydration-dependent phase separation of a prion-like protein regulates seed germination during water stress.

Dundr, M. (2013). Nucleation of nuclear bodies. In Imaging Gene Expression. Totowa, NJ: Humana Press, 351-364.

Emenecker, R. J., Holehouse, A. S., and Strader, L. C. (2020). Emerging roles for phase separation in plants. Dev. Cell 55, 69-83. doi: 10.1016/j.devcel.2020. 09.010

Enderle, B., Sheerin, D. J., Paik, I., Kathare, P. K., Schwenk, P., Klose, C., et al. (2017). PCH1 and PCHL promote photomorphogenesis in plants by controlling phytochrome B dark reversion. Nat. Commun. 8, 1-9.

Fang, X., Wang, L., Ishikawa, R., Li, Y., Fiedler, M., Liu, F., et al. (2019). Arabidopsis FLL2 promotes liquid-liquid phase separation of polyadenylation complexes. Nature 569, 265-269. doi: 10.1038/s41586-019-1165-8

Franklin, K. A., and Quail, P. H. (2010). Phytochrome functions in Arabidopsis development. J. Exp. Bot. 61, 11-24. doi: 10.1093/jxb/erp304

Frohnmeyer, H., and Staiger, D. (2003). Ultraviolet-B radiation-mediated responses in plants. Balancing damage and protection. Plant Physiol. 133, 1420-1428. doi: 10.1104/pp.103.030049

Galvão, R. M., Li, M., Kothadia, S. M., Haskel, J. D., Decker, P. V., Van Buskirk, E. K., et al. (2012). Photoactivated phytochromes interact with HEMERA and promote its accumulation to establish photomorphogenesis in Arabidopsis. Genes Dev. 26, 1851-1863. doi: 10.1101/gad.193219.112

Genoud, T., Schweizer, F., Tscheuschler, A., Debrieux, D., Casal, J. J., Schaf̈er, E., et al. (2008). FHY1 mediates nuclear import of the light-activated phytochrome A photoreceptor. PLoS Genet. 4:e1000143. doi: 10.1371/journal.pgen.100 0143

Gil, P., Kircher, S., Adam, E., Bury, E., Kozma-Bognar, L., Schäfer, E., et al. (2000). Photocontrol of subcellular partitioning of phytochrome-B: GFP fusion protein in tobacco seedlings. Plant J. 22, 135-145. doi: 10.1046/j.1365-313x.2000. 00730.x

Goglia, A. G., and Toettcher, J. E. (2019). A bright future: optogenetics to dissect the spatiotemporal control of cell behavior. Curr. Opin. Chem. Biol. 48, 106-113. doi: 10.1016/j.cbpa.2018.11.010

Gomes, E., and Shorter, J. (2019). The molecular language of membraneless organelles. J. Biol. Chem. 294, 7115-7127. doi: 10.1074/jbc.tm118.001192

Gressel, J. (1979). Blue light photoreception. Photochem. Photobiol. 30, 749-754. doi: 10.1111/j.1751-1097.1979.tb07209.x

Grima, R., Sonntag, S., Venezia, F., Kircher, S., Smith, R. W., and Fleck, C. (2018). Insight into nuclear body formation of phytochromes through stochastic modelling and experiment. Phys. Biol. 15:056003. doi: 10.1088/1478-3975/ aac193 
Gu, N. N., Zhang, Y. C., and Yang, H. Q. (2012). Substitution of a conserved glycine in the PHR domain of Arabidopsis cryptochrome 1 confers a constitutive light response. Mol. Plant 5, 85-97. doi: 10.1093/mp/ss r052

Guo, X., Bulyk, M. L., and Hartemink, A. J. (2012). Intrinsic disorder within and flanking the DNA-binding domains of human transcription factors. Pac. Symp. Biocomput. 2012, 104-115.

Hahm, J., Kim, K., Qiu, Y., and Chen, M. (2020). Increasing ambient temperature progressively disassembles Arabidopsis phytochrome B from individual photobodies with distinct thermostabilities. Nat. Commun. 11, 1-14.

Harmon, T. S., Holehouse, A. S., Rosen, M. K., and Pappu, R. V. (2017). Intrinsically disordered linkers determine the interplay between phase separation and gelation in multivalent proteins. elife 6:e30294.

Helizon, H., Rösler-Dalton, J., Gasch, P., von Horsten, S., Essen, L. O., and Zeidler, M. (2018). Arabidopsis phytochrome A nuclear translocation is mediated by a far-red elongated hypocotyl 1-importin complex. Plant J. 96, 1255-1268. doi: $10.1111 /$ tpj. 14107

Hiltbrunner, A., Tscheuschler, A., Viczia’n, A., Kunkel, T., Kircher, S., and Schafer, E. (2006). FHY1 and FHL act together to mediate nuclear accumulation of the phytochrome A photoreceptor. Plant Cell Physiol. 47, 1023-1034. doi: 10.1093/pcp/pcj087

Hoang, Q. T., Han, Y. J., and Kim, J. I. (2019). Plant phytochromes and their phosphorylation. Int. J. Mol. Sci. 20:3450. doi: 10.3390/ijms20143450

Holehouse, A. S. (2019). "IDPs and IDRs in biomolecular condensates," in Intrinsically disordered proteins, ed. N. Salvi (Cambridge: Academic Press), 209-255. doi: 10.1016/b978-0-12-816348-1.00007-7

Holm, M., Ma, L. G., Qu, L. J., and Deng, X. W. (2002). Two interacting bZIP proteins are direct targets of COP1-mediated control of light-dependent gene ex- pression in Arabidopsis. Genes Dev. 16, 1247-1259. doi: 10.1101/gad. 969702

Hu, W., Franklin, K. A., Sharrock, R. A., Jones, M. A., Harmer, S. L., and Lagarias, J. C. (2013). Unanticipated regulatory roles for Arabidopsis phytochromes revealed by null mutant analysis. Proc. Natl. Acad. Sci. 110, 1542-1547. doi: $10.1073 /$ pnas. 1221738110

Hu, W., Su, Y. S., and Lagarias, J. C. (2009). A light-independent allele of phytochrome B faithfully recapitulates photomorphogenic transcriptional networks. Mol. Plant 2, 166-182. doi: $10.1093 / \mathrm{mp} / \mathrm{ssn} 086$

Huang, H., McLoughlin, K. E., Sorkin, M. L., Burgie, E. S., Bindbeutel, R. K., Vierstra, R. D., et al. (2019). PCH1 regulates light, temperature, and circadian signaling as a structural component of phytochrome B-photobodies in Arabidopsis. Proc. Natl. Acad. Sci. 116, 8603-8608. doi: 10.1073/pnas. 1818217116

Huang, H., Yoo, C. Y., Bindbeutel, R., Goldsworthy, J., Tielking, A., Alvarez, S., et al. (2016). PCH1 integrates circadian and light-signaling pathways to control photoperiod-responsive growth in Arabidopsis. Elife 5:e13292.

Huang, X., Chen, S., Li, W., Tang, L., Zhang, Y., Yang, N., et al. (2021). ROS regulated reversible protein phase separation synchronizes plant flowering. Nat. Chem. Biol. 17, 549-557. doi: 10.1038/s41589-021-00739-0

Huq, E., Al-Sady, B., and Quail, P. H. (2003). Nuclear translocation of the photoreceptor phytochrome B is necessary for its biological function in seedling photomorphogenesis. Plant J. 35, 660-664. doi: 10.1046/j.1365-313x.2003. 01836.x

Hyman, A. A., Weber, C. A., and Jülicher, F. (2014). Liquid-liquid phase separation in biology. Annu. Rev. Cell Dev. Biol. 30, 39-58.

Imaizumi, T., Tran, H. G., Swartz, T. E., Briggs, W. R., and Kay, S. A. (2003). FKF1 is essential for photoperiodic-specific light signalling in Arabidopsis. Nature 426, 302-306. doi: 10.1038/nature02090

Jang, I. C., Henriques, R., Seo, H. S., Nagatani, A., and Chua, N. H. (2010). Arabidopsis PHYTOCHROME INTERACTING FACTOR proteins promote phytochrome B polyubiquitination by COP1 E3 ligase in the nucleus. Plant Cell 22, 2370-2383. doi: 10.1105/tpc.109.072520

Jang, I. C., Yang, S. W., Yang, J. Y., and Chua, N. H. (2007). Independent and interde- pendent functions of LAF1 and HFR1 in phytochrome a signaling. Genes Dev 21, 2100-2111. doi: 10.1101/gad.1568207

Jansen, M. A., Gaba, V., and Greenberg, B. M. (1998). Higher plants and UV$\mathrm{B}$ radiation: balancing damage, repair and acclimation. Trends Plant Sci. 3, 131-135. doi: 10.1016/s1360-1385(98)01215-1
Jarillo, J. A., Gabrys, H., Capel, J., Alonso, J. M., Ecker, J. R., and Cashmore, A. R. (2001). Phototropin-related NPL1 controls chloroplast relocation induced by blue light. Nature 410, 952-954. doi: 10.1038/35073622

Jenkins, G. I., and Brown, B. A. (2018). UV-B perception and signal transduction. Annu. Plant Rev. 30, 155-182. doi: 10.1002/9780470988893.ch7

Jones, A. M., and Quail, P. H. (1989). Phytochrome structure: Peptide fragments from the amino-terminal domain involved in protein-chromophore interactions. Planta 178, 147-156. doi: 10.1007/bf00393189

Jones, M. A. (2009). Entrainment of the Arabidopsis circadian clock. J. Plant Biol. 52, 202-209. doi: 10.1007/s12374-009-9030-1

Jung, J. H., Barbosa, A. D., Hutin, S., Kumita, J. R., Gao, M., Derwort, D., et al. (2020). A prion-like domain in ELF3 functions as a thermosensor in Arabidopsis. Nature 585, 256-260. doi: 10.1038/s41586-020-2644-7

Jung, J. H., Domijan, M., Klose, C., Biswas, S., Ezer, D., Gao, M., et al. (2016). Phytochromes function as thermosensors in Arabidopsis. Science 354, 886-889. doi: $10.1126 /$ science.aaf6005

Kagawa, T., Sakai, T., Suetsugu, N., Oikawa, K., Ishiguro, S., Kato, T., et al. (2001) Arabidopsis NPL1: a phototropin homolog controlling the chloroplast highlight avoidance response. Science 291, 2138-2141. doi: 10.1126/science.291. 5511.2138

Kaiser, T. E., Intine, R. V., and Dundr, M. (2008). De novo formation of a subnuclear body. Science 322, 1713-1717. doi: 10.1126/science.1165216

Kaiserli, E., and Jenkins, G. I. (2007). UV-B promotes rapid nuclear translocation of the Arabidopsis UV-B specific signaling component UVR8 and activates its function in the nucleus. Plant Cell 19, 2662-2673. doi: 10.1105/tpc.107.05 3330

Kaiserli, E., Paldi, K., O’Donnell, L., Batalov, O., Pedmale, U. V., Nusinow, D. A., et al. (2015). Integration of light and photoperiodic signaling in transcriptional nuclear foci. Dev. Cell 35, 311-321. doi: 10.1016/j.devcel.2015.10.008

Kami, C., Lorrain, S., Hornitschek, P., and Fankhauser, C. (2010). Light-regulated plant growth and development. Curr. Top. Dev. Biol. 91, 29-66. doi: 10.1016/ s0070-2153(10)91002-8

Kandori, H., Yoshihara, K., and Tokutomi, S. (1992). Primary process of phytochrome: initial step of photomorphogenesis in green plants. J. Am. Chem. Soc. 114, 10958-10959. doi: 10.1021/ja00053a041

Kasahara, M., Swartz, T. E., Olney, M. A., Onodera, A., Mochizuki, N. Fukuzawa, H., et al. (2002). Photochemical properties of the flavin mononucleotide-binding domains of the phototropins from Arabidopsis, Rice, and Chlamydomonas reinhardtii. Plant Physiol. 129, 762-773. doi: 10.1104/pp. 002410

Kikis, E. A., Oka, Y., Hudson, M. E., Nagatani, A., and Quail, P. H. (2009) Residues clustered in the light-sensing knot of phytochrome B are necessary for conformer-specific binding to signaling partner PIF3. PLoS Genet. 5:e1000352. doi: 10.1371/journal.pgen.1000352

Kim, J. I, Shen, Y., Han, Y. J., Park, J. E., Kirchenbauer, D., Soh, M. S., et al. (2004). Phytochrome phosphorylation modulates light signaling by influencing the protein-protein interaction. Plant Cell 16, 2629-2640. doi: 10.1105/tpc.104. 023879

Kim, L., Kircher, S., Toth, R., Adam, E., Schäfer, E., and Nagy, F. (2000). Lightinduced nuclear import of phytochrome-A: GFP fusion proteins is differentially regulated in transgenic tobacco and Arabidopsis. Plant J. 22, 125-133. doi: 10.1046/j.1365-313x.2000.00729.x

Kim, Y., Lim, J., Yeom, M., Kim, H., Kim, J., Wang, L., et al. (2013). ELF4 regulates GIGANTEA chromatin access through subnuclear sequestration. Cell Rep. 3, 671-677. doi: 10.1016/j.celrep.2013.02.021

Kinoshita, T., Doi, M., Suetsugu, N., Kagawa, T., Wada, M., and Shimazaki, K. I. (2001). Phot 1 and phot 2 mediate blue light regulation of stomatal opening. Nature 414, 656-660. doi: 10.1038/414656a

Kircher, S., Gil, P., Kozma-Bognár, L., Fejes, E., Speth, V., Husselstein-Muller, T., et al. (2002). Nucleocytoplasmic partitioning of the plant photoreceptors phytochrome A, B, C, D, and E is regulated differentially by light and exhibits a diurnal rhythm. Plant Cell 14, 1541-1555. doi: 10.1105/tpc.00 1156

Kircher, S., Kozma-Bognar, L., Kim, L., Adam, E., Harter, K., Schäfer, E., et al. (1999). Light quality-dependent nuclear import of the plant photoreceptors phytochrome A and B. Plant Cell 11, 1445-1456. doi: 10.2307/38 70974 
Kleine, T., Lockhart, P., and Batschauer, A. (2003). An Arabidopsis protein closely related to Synechocystis cryptochrome is targeted to organelles. Plant J. 35, 93-103. doi: 10.1046/j.1365-313x.2003.01787.x

Klose, C., Nagy, F., and Schäfer, E. (2020). Thermal reversion of plant phytochromes. Mol. Plant 13, 386-397. doi: 10.1016/j.molp.2019.12.004

Klose, C., Venezia, F., Hussong, A., Kircher, S., Schäfer, E., and Fleck, C. (2015a). Systematic analysis of how phytochrome B dimerization determines its specificity. Nat. Plants 1, 1-9.

Klose, C., Viczián, A., Kircher, S., Schäfer, E., and Nagy, F. (2015b). Molecular mechanisms for mediating light-dependent nucleo/cytoplasmic partitioning of phytochrome photoreceptors. New Phytol. 206, 965-971. doi: 10.1111/nph. 13207

Krall, L., and Reed, J. W. (2000). The histidine kinase-related domain participates in phytochrome B function but is dispensable. Proc. Natl. Acad. Sci. 97, 8169-8174. doi: 10.1073/pnas.140520097

Lallemand-Breitenbach, V. (2010). PML nuclear bodies. Cold Spring Harb. Perspect. Biol. 2:a000661.

Legris, M., Klose, C., Burgie, E. S., Rojas, C. C. R., Neme, M., Hiltbrunner, A., et al. (2016). Phytochrome B integrates light and temperature signals in Arabidopsis. Science 354, 897-900. doi: 10.1126/science.aaf5656

Leivar, P., and Quail, P. H. (2011). PIFs: pivotal components in a cellular signaling hub. Trends Plant Sci. 16, 19-28. doi: 10.1016/j.tplants.2010.08.003

Leivar, P., Tepperman, J. M., Monte, E., Calderon, R. H., Liu, T. L., and Quail, P. H. (2009). Definition of early transcriptional circuitry involved in lightinduced reversal of PIF-imposed repression of photomorphogenesis in young Arabidopsis seedlings. Plant Cell 21, 3535-3553. doi: 10.1105/tpc.109.070672

Lian, H. L., He, S. B., Zhang, Y. C., Zhu, D. M., Zhang, J. Y., Jia, K. P., et al. (2011). Blue-light-dependent interaction of cryptochrome 1 with SPA1 defines a dynamic signaling mechanism. Genes Dev. 25, 1023-1028. doi: 10.1101/gad. 2025111

Lin, C. (2000). Plant blue-light receptors. Trends Plant Sci. 5, 337-342. doi: 10. 1016/s1360-1385(00)01687-3

Lin, C. (2002). Blue light receptors and signal transduction. Plant Cell 14, S207S225.

Liu, B., Zuo, Z., Liu, H., Liu, X., and Lin, C. (2011). Arabidopsis cryptochrome 1 interacts with SPA1 to suppress COP1 activity in response to blue light. Genes Dev. 25, 1029-1034.

Liu, Q., Wang, Q., Deng, W., Wang, X., Piao, M., Cai, D., et al. (2017). Molecular basis for blue light-dependent phosphorylation of Arabidopsis cryptochrome 2. Nat. Commun. 8, 1-12.

Liu, Y., Liu, Q., Yan, Q., Shi, L., and Fang, Y. (2014). Nucleolus-tethering system (NoTS) reveals that assembly of photobodies follows a self-organization model. Mol. Biol. Cell. 25, 1366-1373. doi: 10.1091/mbc.e13-09-0527

López-Juez, E., Nagatani, A., Tomizawa, K. L., Deak, M., Kern, R., Kendrick, R. E., et al. (1992). The cucumber long hypocotyl mutant lacks a light-stable PHYB-like phytochrome. Plant Cell 4, 241-251. doi: 10.2307/3869536

Lorrain, S., Allen, T., Duek, P. D., Whitelam, G. C., and Fankhauser, C. (2008). Phytochrome-mediated inhibition of shade avoidance involves degradation of growth-promoting bHLH transcription factors. Plant J. 53, 312-323. doi: 10. 1111/j.1365-313x.2007.03341.x

Lorrain, S., Trevisan, M., Pradervand, S., and Fankhauser, C. (2009). Phytochrome interacting factors 4 and 5 redundantly limit seedling de-etiolation in continuous far-red light. Plant J. 60, 449-461. doi: 10.1111/j.1365-313x.2009. 03971.x

Lu, X. D., Zhou, C. M., Xu, P. B., Luo, Q., Lian, H. L., and Yang, H. Q. (2015). Red-light-dependent interaction of phyB with SPA1 promotes COP1-SPA1 dissociation and photomorphogenic development in Arabidopsis. Mol. Plant 8, 467-478. doi: 10.1016/j.molp.2014.11.025

Mao, Y. S., Zhang, B., and Spector, D. L. (2011). Biogenesis and function of nuclear bodies. Trends Genet. 27, 295-306. doi: 10.1016/j.tig.2011.05.006

Martin, E. W., and Holehouse, A. S. (2020). Intrinsically disordered protein regions and phase separation: sequence determinants of assembly or lack thereof. Emerg. Top. Life Sci. 4, 307-329. doi: 10.1042/etls20190164

Martínez-García, J. F., Huq, E., and Quail, P. H. (2000). Direct targeting of light signals to a promoter element-bound transcription factor. Science 288, 859-863. doi: $10.1126 /$ science.288.5467.859

Mathews, S. (2010). Evolutionary studies illuminate the structural-functional model of plant phytochromes. Plant Cell 22, 4-16. doi: 10.1105/tpc.109.072280
Matsushita, T., Mochizuki, N., and Nagatani, A. (2003). Dimers of the N-terminal domain of phytochrome B are functional in the nucleus. Nature 424, 571-574. doi: 10.1038/nature 01837

McCormac, A. C., Wagner, D., Boylan, M. T., Quail, P. H., Smith, H., and Whitelam, G. C. (1993). Photoresponses of transgenic Arabidopsis seedlings expressing introduced phytochrome B-encoding cDNAs: evidence that phytochrome A and phytochrome B have distinct photoregulatory functions. Plant J. 4, 19-27. doi: 10.1046/j.1365-313x.1993.04010019.x

Medzihradszky, M., Bindics, J., Ádám, É, Viczián, A., Klement, É, Lorrain, S., et al. (2013). Phosphorylation of phytochrome B inhibits light-induced signaling via accelerated dark reversion in Arabidopsis. Plant Cell 25, 535-544. doi: 10.1105/ tpc. 112.106898

Meyer, H. M. (2020). In search of function: nuclear bodies and their possible roles as plant environmental sensors. Curr. Opin. Plant Biol. 58, 33-40. doi: 10.1016/j.pbi.2020.10.002

Monneron, A., and Bernhard, W. (1969). Fine structural organization of the interphase nucleus in some mammalian cells. J. Ultrastruct. Res. 27, 266-288. doi: 10.1016/s0022-5320(69)80017-1

Murcia, G., Enderle, B., Hiltbrunner, A., and Casal, J. J. (2020). Phytochrome B and $\mathrm{PCH} 1$ protein dynamics store night temperature information. Plant J. 105, 22-33. doi: 10.1111/tpj.15034

Nagatani, A. (2010). Phytochrome: structural basis for its functions. Curr. Opin. Plant Biol. 13, 565-570. doi: 10.1016/j.pbi.2010.07.002

Nagatani, A., Reed, J. W., and Chory, J. (1993). Isolation and initial characterization of Arabidopsis mutants that are deficient in phytochrome A. Plant Physiol. 102, 269-277. doi: 10.1104/pp.102.1.269

Nagy, F., and Schäfer, E. (2002). Phytochromes control photomorphogenesis by differentially regulated, interacting signaling pathways in higher plants. Annu. Rev. Plant Biol. 53, 329-355.

Nelson, D. C., Lasswell, J., Rogg, L. E., Cohen, M. A., and Bartel, B. (2000). FKF1, a clock- controlled gene that regulates the transition to flowering in Arabidopsis. Cell 101, 331-340. doi: 10.1016/s0092-8674(00) 80842-9

Nevarez, P. A., Qiu, Y., Inoue, H., Yoo, C. Y., Benfey, P. N., Schnell, D. J., et al. (2017). Mechanism of dual targeting of the phytochrome signaling component HEMERA/pTAC12 to plastids and the nucleus. Plant Physiol. 173, 1953-1966. doi: $10.1104 /$ pp.16.00116

Ni, M., Tepperman, J. M., and Quail, P. H. (1998). PIF3, a phytochrome-interacting factor necessary for normal photoinduced signal transduction, is a novel basic helix-loop-helix protein. Cell 95, 657-667. doi: 10.1016/s0092-8674(00)81 636-0

Ni, W., Xu, S. L., Chalkley, R. J., Pham, T. N. D., Guan, S., Maltby, D. A., et al. (2013). Multisite light-induced phosphorylation of the transcription factor PIF3 is necessary for both its rapid degradation and concomitant negative feedback modulation of photoreceptor phyB levels in Arabidopsis. Plant Cell 25, 2679-2698. doi: 10.1105/tpc.113. 112342

Ni, W., Xu, S. L., Tepperman, J. M., Stanley, D. J., Maltby, D. A., Gross, J. D., et al. (2014). A mutually assured destruction mechanism attenuates light signaling in Arabidopsis. Science 344, 1160-1164. doi: 10.1126/science.1250778

Oh, E., Yamaguchi, S., Hu, J., Yusuke, J., Jung, B., Paik, I., et al. (2007). PIL5, a phytochrome-interacting bHLH protein, regulates gibberellin responsiveness by binding directly to the GAI and RGA pro- moters in Arabidopsis seeds. Plant Cell 19, 1192-1208. doi: 10.1105/tpc.107.05 0153

Oka, Y., Matsushita, T., Mochizuki, N., Quail, P. H., and Nagatani, A. (2008). Mutant screen distinguishes between residues necessary for light-signal perception and signal transfer by phytochrome B. PLoS Genet. 4:e1000158. doi: 10.1371/journal.pgen.1000158

Ouyang, M., Li, X., Zhang, J., Feng, P., Pu, H., Kong, L., et al. (2020). Liquid-liquid phase transition drives intra-chloroplast cargo sorting. Cell 180, 1144-1159. doi: 10.1016/j.cell.2020.02.045

Owen, I., and Shewmaker, F. (2019). The role of posttranslational modifications in the phase transitions of intrinsically disordered proteins. Int. J. Mol. Sci. 20:5501. doi: 10.3390/ijms20215501

Paik, I., and Huq, E. (2019). Plant photoreceptors: Multi-functional sensory proteins and their signaling networks. Semin. Cell Dev. Biol. 92, 114-121. doi: 10.1016/j.semcdb.2019.03.007 
Parks, B. M., and Quail, P. H. (1993). Hy8, a new class of Arabidopsis long hypocotyl mutants deficient in functional phytochrome A. Plant Cell 5, 39-48. doi: $10.2307 / 3869426$

Perrella, G., Zioutopoulou, A., Hamilton, A., and Kaiserli, E. (2021). Photobody Detection Using Immunofluorescence and Super-Resolution Imaging in Arabidopsis. Methods Mol. Biol. (Clifton, NJ) 2297, 7-19. doi: 10.1007/978-10716-1370-2_2

Pfalz, J., Liere, K., Kandlbinder, A., Dietz, K. J., and Oelmüller, R. (2006). pTAC2,6, and-12 are components of the transcriptionally active plastid chromosome that are required for plastid gene expression. Plant Cell 18, 176-197. doi: 10.1105/tpc.105.036392

Pfeiffer, A., Nagel, M. K., Popp, C., Wüst, F., Bindics, J., Viczián, A., et al. (2012). Interaction with plant transcription factors can mediate nuclear import of phytochrome B. Proc. Natl. Acad. Sci. 109, 5892-5897. doi: 10.1073/pnas. 1120764109

Posey, A. E., Holehouse, A. S., and Pappu, R. V. (2018). Phase separation of intrinsically disordered proteins. Methods Enzymol. 611, 1-30. doi: 10.1016/ bs.mie.2018.09.035

Powers, S. K., Holehouse, A. S., Korasick, D. A., Schreiber, K. H., Clark, N. M., Jing, H., et al. (2019). Nucleo-cytoplasmic partitioning of ARF proteins controls auxin responses in Arabidopsis thaliana. Mol. Cell 76, 177-190. doi: 10.1016/j. molcel.2019.06.044

Qiu, Y., Li, M., Jean, R., Moore, C. M., and Chen, M. (2019). Daytime temperature is sensed by phytochrome $\mathrm{B}$ in Arabidopsis through a transcriptional activator HEMERA. Nat. Commun. 10, 1-13.

Qiu, Y., Li, M., Pasoreck, E. K., Long, L., Shi, Y., Galvão, R. M., et al. (2015). HEMERA couples the proteolysis and transcriptional activity of PHYTOCHROME INTERACTING FACTORs in Arabidopsis photomorphogenesis. Plant Cell 27, 1409-1427. doi: 10.1105/tpc.114.13 6093

Qiu, Y., Pasoreck, E. K., Reddy, A. K., Nagatani, A., Ma, W., Chory, J., et al. (2017). Mechanism of early light signaling by the carboxy-terminal output module of Arabidopsis phytochrome B. Nat. Commun. 8, 1-17.

Qu, G. P., Li, H., Lin, X. L., Kong, X., Hu, Z. L., Jin, Y. H., et al. (2020). Reversible SUMOylation of FHY1 regulates Phytochrome A signaling in Arabidopsis. Mol. Plant 13, 879-893. doi: 10.1016/j.molp.2020.04.002

Quail, P. H. (1991). Phytochrome: a light-activated molecular switch that regulates plant gene expression. Annu. Rev. Genet. 25, 389-409. doi: 10.1146/annurev.ge. 25.120191.002133

Quail, P. H. (2010). Phytochromes. Curr. Biol. 20, R504-R507.

Quail, P. H., Briggs, W. R., and Pratt, L. H. (1978). In vivo phosphorylation of phytochrome. Carnegie Instit. Yearbook 77, 342-344.

Rausenberger, J., Hussong, A., Kircher, S., Kirchenbauer, D., Timmer, J., Nagy, F., et al. (2010). An integrative model for phytochrome B mediated photomorphogenesis: from protein dynamics to physiology. PLoS One 5:e10721. doi: 10.1371/journal.pone.0010721

Reed, J. W., Nagatani, A., Elich, T. D., Fagan, M., and Chory, J. (1994). Phytochrome A and phytochrome B have overlapping but distinct functions in Arabidopsis development. Plant Physiol. 104, 1139-1149. doi: 10.1104/pp.104. 4.1139

Rizzini, L., Favory, J. J., Cloix, C., Faggionato, D., O’Hara, A., Kaiserli, E., et al. (2011). Perception of UV-B by the Arabidopsis UVR8 protein. Science 332, 103-106.

Rockwell, N. C., Su, Y. S., and Lagarias, J. C. (2006). Phytochrome structure and signaling mechanisms. Annu. Rev. Plant Biol. 57, 837-858. doi: 10.1146/ annurev.arplant.56.032604.144208

Roden, C., and Gladfelter, A. S. (2021). RNA contributions to the form and function of biomolecular condensates. Nat. Rev. Mol. Cell Biol. 22, 183-195. doi: 10.1038/s41580-020-0264-6

Ronald, J., and Davis, S. J. (2019). Focusing on the nuclear and subnuclear dynamics of light and circadian signalling. Plant Cell Environ. 42, 2871-2884. doi: $10.1111 /$ pce. 13634

Sabari, B. R., Dall'Agnese, A., and Young, R. A. (2020). Biomolecular condensates in the nucleus. Trends Biochem. Sci. 45, 961-977. doi: 10.1016/j.tibs.2020.06.007

Sadanandom, A., Ádám, É, Orosa, B., Viczián, A., Klose, C., Zhang, C., et al. (2015). SUMOylation of phytochrome-B negatively regulates light-induced signaling in Arabidopsis thaliana. Proc. Natl. Acad. Sci. 112, 11108-11113. doi: 10.1073/ pnas. 1415260112
Sakai, T., Kagawa, T., Kasahara, M., Swartz, T. E., Christie, J. M., Briggs, W. R., et al. (2001). Arabidopsis nph1 and npl1: blue light receptors that mediate both phototropism and chloroplast relocation. Proc Natl. Acad. Sci. 98, 6969-6974. doi: 10.1073/pnas.101137598

Sakamoto, K., and Nagatani, A. (1996). Nuclear localization activity of phytochrome B. Plant J. 10, 859-868. doi: 10.1046/j.1365-313x.1996.10050 859.x

Schneider, N., Wieland, F. G., Kong, D., Fischer, A. A., Hörner, M., Timmer, J., et al. (2021). Liquid-liquid phase separation of light-inducible transcription factors increases transcription activation in mammalian cells and mice. Sci. Adv. 7:eabd3568. doi: 10.1126/sciadv.abd3568

Schultz, T. F., Kiyosue, T., Yanovsky, M., Wada, M., and Kay, S. A. (2001). A role for LKP2 in the circadian clock of Arabidopsis. Plant Cell 13, 2659-2670. doi: 10.1105/tpc.13.12.2659

Semenov, A. N., and Rubinstein, M. (1998). Thermoreversible gelation in solutions of associative polymers. 1. Stat. Macromol. 31, 1373-1385. doi: 10.1021/ ma970616h

Shalitin, D., Yu, X., Maymon, M., Mockler, T., and Lin, C. (2003). Blue lightdependent in vivo and in vitro phosphorylation of Arabidopsis cryptochrome 1. Plant Cell 15, 2421-2429. doi: 10.1105/tpc.013011

Sharrock, R. A., and Clack, T. (2004). Heterodimerization of type II phytochromes in Arabidopsis. Proc. Natl. Acad. Sci. 101, 11500-11505. doi: 10.1073/pnas. 0404286101

Sheerin, D. J., Menon, C., zur Oven-Krockhaus, S., Enderle, B., Zhu, L., Johnen, P., et al. (2015). Light-activated phytochrome A and B interact with members of the SPA family to promote photomorphogenesis in Arabidopsis by reorganizing the COP1/SPA complex. Plant Cell 27, 189-201. doi: 10.1105/tpc.114.134775

Shen, H., Zhu, L., Castillon, A., Majee, M., Downie, B., and Huq, E. (2008). Light- induced phosphorylation and degradation of the negative regulator PHYTOCHROME-INTERACTING FACTOR1 from Arabidopsis depend upon its direct physical interactions with photoactivated phytochromes. Plant Cell 20, 1586-1602. doi: 10.1105/tpc.108.060020

Shevtsov, S. P., and Dundr, M. (2011). Nucleation of nuclear bodies by RNA. Nat. Cell Biol. 13, 167-173. doi: 10.1038/ncb2157

Shikata, H., Hanada, K., Ushijima, T., Nakashima, M., Suzuki, Y., and Matsushita, T. (2014). Phytochrome controls alternative splicing to mediate light responses in Arabidopsis. Proc. Natl. Acad. Sci. 111, 18781-18786. doi: 10.1073/pnas. 1407147112

Shimizu-Sato, S., Huq, E., Tepperman, J. M., and Quail, P. H. (2002). A lightswitchable gene promoter system. Nat. Biotechnol. 20, 1041-1044. doi: 10.1038/ nbt734

Shin, J., Kim, K., Kang, H., Zulfugarov, I. S., Bae, G., Lee, C. H., et al. (2009). Phytochromes promote seedling light responses by inhibiting four negativelyacting phytochrome-interacting factors. Proc. Natl. Acad. Sci. USA. 106, 76607665. doi: 10.1073/pnas.0812219106

Somers, D. E., Devlin, P. F., and Kay, S. A. (1998). Phytochromes and cryptochromes in the entrainment of the Arabidopsis circadian clock. Science 282, 1488-1490. doi: 10.1126/science.282.5393.1488

Somers, D. E., Schultz, T. F., Milnamow, M., and Kay, S. A. (2000). ZEITLUPE encodes a novel clock-associated PAS protein from Arabidopsis. Cell 101, 319-329. doi: 10.1016/s0092-8674(00)80841-7

Somers, D. E., Sharrock, R. A., Tepperman, J. M., and Quail, P. H. (1991). The hy3 long hypocotyl mutant of Arabidopsis is deficient in phytochrome B. Plant Cell 3, 1263-1274. doi: 10.1105/tpc.3.12.1263

Spannl, S., Tereshchenko, M., Mastromarco, G. J., Ihn, S. J., and Lee, H. O. (2019). Biomolecular condensates in neurodegeneration and cancer. Traffic 20, 890-911. doi: 10.1111/tra.12704

Spector, D. L. (2006). SnapShot: cellular bodies. Cell 127:1071.

Srivastava, M., Sadanandom, A., and Srivastava, A. K. (2021). Towards understanding the multifaceted role of SUMOylation in plant growth and development. Physiol. Plantarum 171, 77-85. doi: 10.1111/ppl. 13204

Steiner, S., Schröter, Y., Pfalz, J., and Pfannschmidt, T. (2011). Identification of essential subunits in the plastid-encoded RNA polymerase complex reveals building blocks for proper plastid development. Plant Physiol. 157, 1043-1055. doi: 10.1104/pp.111.184515

Stuurman, N., Meijne, A. M., van der Pol, A. J., de Jong, L., van Driel, R., and Van Renswoude, J. (1990). The nuclear matrix from cells of different origin. 
Evidence for a common set of matrix proteins. J. Biol. Chem. 265, 5460-5465. doi: 10.1016/s0021-9258(19)39383-4

$\mathrm{Su}$, Y. S., and Lagarias, J. C. (2007). Light-independent phytochrome signaling mediated by dominant GAF domain tyrosine mutants of Arabidopsis phytochromes in transgenic plants. Plant Cell 19, 2124-2139. doi: 10.1105/tpc. 107.051516

Subramanian, C., Kim, B. H., Lyssenko, N. N., Xu, X., Johnson, C. H., and von Arnim, A. G. (2004). The Arabidopsis repressor of light signaling, COP1, is regu- lated by nuclear exclusion: mutational analysis by bioluminescence resonance energy transfer. Proc. Natl. Acad. Sci. USA. 101, 6798-6802. doi: 10.1073/pnas.0307964101

Taslimi, A., Vrana, J. D., Chen, D., Borinskaya, S., Mayer, B. J., Kennedy, M. J., et al. (2014). An optimized optogenetic clustering tool for probing protein interaction and function. Nat. Commun. 5, 1-9.

Terry, M. J., McDowell, M. T., and Lagarias, J. C. (1995). (3Z)-and (3E)phytochromobilin are intermediates in the biosynthesis of the phytochrome chromophore. J. Biol. Chem. 270, 11111-11118. doi: 10.1074/jbc.270.19.11111

Trinkle-Mulcahy, L., and Sleeman, J. E. (2017). The Cajal body and the nucleolus:"In a relationship" or "It's complicated"? RNA Biol. 14, 739-751. doi: $10.1080 / 15476286.2016 .1236169$

Trupkin, S. A., Legris, M., Buchovsky, A. S., Rivero, M. B. T., and Casal, J. J. (2014). Phytochrome B nuclear bodies respond to the low red to far-red ratio and to the reduced irradiance of canopy shade in Arabidopsis. Plant Physiol. 165, 1698-1708. doi: 10.1104/pp.114.242438

Ulm, R., and Nagy, F. (2005). Signalling and gene regulation in response to ultraviolet light. Curr. Opin. Plant Biol. 8, 477-482. doi: 10.1016/j.pbi.2005. 07.004

Ushijima, T., Hanada, K., Gotoh, E., Yamori, W., Kodama, Y., Tanaka, H., et al. (2017). Light controls protein localization through phytochrome-mediated alternative promoter selection. Cell 171, 1316-1325. doi: 10.1016/j.cell.2017. 10.018

Van Buskirk, E. K., Decker, P. V., and Chen, M. (2012). Photobodies in light signaling. Plant Physiol. 158, 52-60. doi: 10.1104/pp.111.186411

Van Buskirk, E. K., Reddy, A. K., Nagatani, A., and Chen, M. (2014). Photobody localization of phytochrome B is tightly correlated with prolonged and lightdependent inhibition of hypocotyl elongation in the dark. Plant Physiol. 165, 595-607. doi: 10.1104/pp.114.236661

Wagner, D., Koloszvari, M., and Quail, P. H. (1996). Two small spatially distinct regions of phytochrome B are required for efficient signaling rates. Plant Cell 8 , 859-871. doi: $10.2307 / 3870288$

Wang, A., Zhang, Z., and Li, G. (2018). Higher accuracy achieved in the simulations of protein structure refinement, protein folding, and intrinsically disordered proteins using polarizable force fields. J. Phys. Chem. Lett. 9, 7110-7116. doi: 10.1021/acs.jpclett.8b03471

Wang, H., Ma, L. G., Li, J. M., Zhao, H. Y., and Deng, X. W. (2001). Direct interaction of Arabidopsis cryptochromes with COP1 in light control development. Science 294, 154-158. doi: 10.1126/science.1063630

Wang, J., Du, X., Pan, W., Wang, X., and Wu, W. (2015). Photoactivation of the cryptochrome/photolyase superfamily. J. Photochem. Photobiol. C Photochem. Rev. 22, 84-102. doi: 10.1016/j.jphotochemrev.2014.12.001

Wang, Q., Zuo, Z., Wang, X., Liu, Q., Gu, L., Oka, Y., et al. (2018). Beyond the photocycle-how cryptochromes regulate photoresponses in plants? Curr. Opin. Plant Biol. 45, 120-126. doi: 10.1016/j.pbi.2018.05.014

Whitelam, G. C., Johnson, E., Peng, J., Carol, P., Anderson, M. L., Cowl, J. S., et al. (1993). Phytochrome a null mutants of Arabidopsis display a wild-type phenotype in white light. Plant Cell 5, 757-768. doi: 10.2307/3869613

Whitelam, G. C., McCormac, A. C., Boylan, M. T., and Quail, P. H. (1992). Photoresponses of Arabidopsis seedlings expressing an introduced oat phyA cDNA: persistence of etiolated plant type responses in light-grown plants. Photochem. Photobiol. 56, 617-621. doi: 10.1111/j.1751-1097.1992.tb02211.x

Wilkinson, E. G., and Strader, L. C. (2020). A Prion-based thermosensor in plants. Mol. Cell. 80, 181-182. doi: 10.1016/j.molcel.2020.09.026
Xin, R., Zhu, L., Salomé, P. A., Mancini, E., Marshall, C. M., Harmon, F. G., et al. (2017). SPF45-related splicing factor for phytochrome signaling promotes photomorphogenesis by regulating pre-mRNA splicing in Arabidopsis. Proc. Natl. Acad. Sci. 114, E7018-E7027.

Yamaguchi, R., Nakamura, M., Mochizuki, N., Kay, S. A., and Nagatani, A. (1999). Light-dependent translocation of a phytochrome B-GFP fusion protein to the nucleus in transgenic Arabidopsis. J. Cell Biol. 145, 437-445. doi: 10.1083/jcb. 145.3.437

Yan, H., Marquardt, K., Indorf, M., Jutt, D., Kircher, S., Neuhaus, G., et al. (2011). Nuclear localization and interaction with COP1 are required for STO/BBX24 function during photomorphogenesis. Plant Physiol. 156, 1772-1782. doi: 10. 1104/pp.111.180208

Yang, E. J., Yoo, C. Y., Liu, J., Wang, H., Cao, J., Li, F. W., et al. (2019). NCP activates chloroplast transcription by controlling phytochrome-dependent dual nuclear and plastidial switches. Nat. Commun. 10, 1-13.

Yoo, C. Y., Pasoreck, E. K., Wang, H., Cao, J., Blaha, G. M., Weigel, D., et al. (2019). Phytochrome activates the plastid-encoded RNA polymerase for chloroplast biogenesis via nucleus-to-plastid signaling. Nat. Commun. 10, 1-16.

Yoo, H., Triandafillou, C., and Drummond, D. A. (2019). Cellular sensing by phase separation: Using the process, not just the products. J. Biol. Chem. 294, 7151-7159. doi: 10.1074/jbc.tm118.001191

Yu, J. W., Rubio, V., Lee, N. Y., Bai, S., Lee, S. Y., Kim, S. S., et al. (2008). COP1 and ELF3 control circadian function and photoperiodic flowering by regulating GI stability. Mol. Cell. 32, 617-630. doi: 10.1016/j.molcel.2008.09.026

Yu, X., Klejnot, J., Zhao, X., Shalitin, D., Maymon, M., Yang, H., et al. (2007). Arabidopsis cryptochrome 2 completes its posttranslational life cycle in the nucleus. Plant Cell 19, 3146-3156. doi: 10.1105/tpc.107.053017

Yu, X., Liu, H., Klejnot, J., and Lin, C. (2010). The cryptochrome blue light receptors. Arabidopsis Book 8:e0135. doi: 10.1199/tab.0135

Yu, X., Sayegh, R., Maymon, M., Warpeha, K., Klejnot, J., Yang, H., et al. (2009). Formation of nuclear bodies of Arabidopsis CRY2 in response to blue light is associated with its blue light-dependent degradation. Plant Cell 21, 118-130. doi: $10.1105 /$ tpc. 108.061663

Zavaliev, R., Mohan, R., Chen, T., and Dong, X. (2020). Formation of NPR1 condensates promotes cell survival during the plant immune response. Cell 182, 1093-1108. doi: 10.1016/j.cell.2020.07.016

Zheng, X., Wu, S., Zhai, H., Zhou, P., Song, M., Su, L., et al. (2013). Arabidopsis phytochrome B promotes SPA1 nuclear accumulation to repress photomorphogenesis under far-red light. Plant Cell 25, 115-133. doi: 10.1105/ tpc.112.107086

Zuo, Z., Liu, H., Liu, B., Liu, X., and Lin, C. (2011). Blue light-dependent interaction of CRY2 with SPA1 regulates COP1 activity and floral initiation in Arabidopsis. Curr. Biol. 21, 841-847. doi: 10.1016/j.cub.2011.03.048

Conflict of Interest: The authors declare that the research was conducted in the absence of any commercial or financial relationships that could be construed as a potential conflict of interest.

The handling editor declared a past co-authorship with one of the author DN.

Publisher's Note: All claims expressed in this article are solely those of the authors and do not necessarily represent those of their affiliated organizations, or those of the publisher, the editors and the reviewers. Any product that may be evaluated in this article, or claim that may be made by its manufacturer, is not guaranteed or endorsed by the publisher.

Copyright (c) 2021 Pardi and Nusinow. This is an open-access article distributed under the terms of the Creative Commons Attribution License (CC BY). The use, distribution or reproduction in other forums is permitted, provided the original author(s) and the copyright owner(s) are credited and that the original publication in this journal is cited, in accordance with accepted academic practice. No use, distribution or reproduction is permitted which does not comply with these terms. 